\title{
Stability in linear optimization under perturbations of the left-hand side coefficients
}

\author{
A. Danillidis, M.A. Goberna, M.A. Lopez, R. Lucchetti
}

\begin{abstract}
This paper studies stability properties of linear optimization problems with finitely many variables and an arbitrary number of constraints, when only left hand side coefficients can be perturbed. The coefficients of the constraints are assumed to be continuous functions with respect to an index which ranges on certain compact Hausdorff topological space, and these properties are preserved by the admissible perturbations. More in detail, the paper analyzes the continuity properties of the feasible set, the optimal set and the optimal value, as well as the preservation of desirable properties (boundednesss, uniqueness) of the feasible and of the optimal sets, under sufficiently small perturbations.
\end{abstract}

Mathematics Subject Classification (2000):

Primary: 49K40, 90C34; Secondary: 15A39, 90C05.

Key words: Stability, linear optimization, semi-infinite optimization.

\section{Introduction}

Let $T$ be a compact Hausdorff topological space (a particular instance being a finite set equipped with the discrete topology), $b \in \mathcal{C}(T, \mathbb{R})$ and $c \in \mathbb{R}^{n} \backslash\left\{\mathbf{0}_{n}\right\}$. We consider the parametric problem

$$
\begin{aligned}
& P(a): \text { inf } c^{\prime} x \\
& \text { s.t. } \quad a_{t}^{\prime} x \geq b_{t}, t \in T \text {. }
\end{aligned}
$$

To this family of problems, depending on the parameter $a=\left\{a_{t}\right\}_{t \in T}$ ranging on $\mathcal{C}\left(T, \mathbb{R}^{n}\right)$, equipped with the supremum norm $\|\cdot\|_{\infty}$, we attach the following sets/values:

1. The feasible set $\mathcal{F}(a)$, i.e. the set of all $x \in \mathbb{R}^{n}$ such that $a_{t}^{\prime} x \geq b_{t}, t \in T$;

2. The optimal set $\mathcal{S}(a)$, i.e. the set of all $x \in \mathbb{R}^{n}$ minimizing $c^{\prime} x$ on $\mathcal{F}(a)$;

3. The optimal value $v(a)$ for all $a \in \mathcal{C}\left(T, \mathbb{R}^{n}\right)$, with $v(a)=+\infty$ whenever $\mathcal{F}(a)=\emptyset$, and $v(a)=-\infty$ if $c^{\prime} x$ is unbounded below on $\mathcal{F}(a)$.

Thus, $\mathcal{F}, \mathcal{S}: \mathcal{C}\left(T, \mathbb{R}^{n}\right) \rightrightarrows \mathbb{R}^{n}$ are set-valued mappings whose domains, denoted by $\operatorname{dom} \mathcal{F}$ and $\operatorname{dom} \mathcal{S}$, are the sets of those $a \in \mathcal{C}\left(T, \mathbb{R}^{n}\right)$ such that $\mathcal{F}(a)$ and $\mathcal{S}(a)$ are nonempty, respectively. The domain of the ordinary mapping $v: \mathcal{C}\left(T, \mathbb{R}^{n}\right) \rightarrow \mathbb{R} \cup\{ \pm \infty\}$, i.e. the set of those $a$ such that $v(a)<+\infty$, obviously coincides with $\operatorname{dom} \mathcal{F}$. We shall also consider the following sets:

1. $\mathcal{B}_{\mathcal{F}}$ is the set of parameters $a$ such that $\mathcal{F}(a)$ is nonempty and bounded;

2. $\mathcal{U}_{\mathcal{F}}$ is the set of parameters $a$ such that $\mathcal{F}(a)$ is a singleton;

3. $\mathcal{B}_{\mathcal{S}}$ is the set of parameters $a$ such that $\mathcal{S}(a)$ is nonempty and bounded; 
4. $\mathcal{U}_{\mathcal{S}}$ is the set of parameters $a$ such that $\mathcal{S}(a)$ is a singleton.

In this paper we are mainly interested in the description of relevant topological and continuity properties of $\mathcal{F}, \mathcal{S}$ and $v$. In particular, about the issue of continuity, we focus on upper/lower semicontinuity properties of $\mathcal{F}, \mathcal{S}$ with respect to perturbations. We aim at characterizing parameters $a \in \mathcal{C}\left(T, \mathbb{R}^{n}\right)$ for which properties like boundedness of feasible/optimal set remain invariant under small perturbations. This question can be reformulated in terms of characterizing the topological interior of the sets $\mathcal{B}_{\mathcal{F}}, \mathcal{U}_{\mathcal{F}}, \mathcal{B}_{\mathcal{S}}$ and $\mathcal{U}_{\mathcal{S}}$. A more general approach would consist in considering parameters $a$ ranging on a subset $\Omega$ of $\mathcal{C}\left(T, \mathbb{R}^{n}\right)$, that is, restricting the set of allowed perturbations, see Example 1.2. In this work we shall only consider the case $\Omega=\mathcal{C}\left(T, \mathbb{R}^{n}\right)$.

Let us also point out that we consider only left-hand side perturbations, in the sense that vectors $c$ (objective function) and $b$ (right-hand side coefficients) are kept fixed in this setting. This ostensibly innocent detail eventually increases the difficulty of the study. The same phenomenon arises in sensitivity analysis in linear programming, whose objective consists in estimating the impact on the optimal value of perturbing the data: there exists a wide literature on perturbations of $b$ and $c$ (see for instance [16] for right-hand side perturbations), but few works are devoted to analyze only left-hand side perturbations; the interest of the latter is illustrated by the following examples.

Example 1.1 (finite zero-sum game). This first example comes from game theory, more precisely the setting of two person, finite, zero sum games. They are described by a real valued matrix $P$, player I chooses a row $i$ player II chooses a column $j$ and the resulting pay-off $p_{i j}$ is what the second player pays (in algebraic sense) to the first. Thus player I in a sense tries to get the maximum possible, while the second one wishes to minimize payments. The celebrated minimax theorem of von Neumann states that such games have (Nash) equilibria in mixed strategies. Furthermore, Player II can select an optimal strategy by solving the auxiliary problem (by assuming, without loss of generality $p_{i j}>0$ for all $i, j$ )

$$
\begin{aligned}
& P(a): \text { inf } \mathbf{1}^{\prime} x \\
& \text { s.t. } P^{\prime} x \geq 1 \text {, }
\end{aligned}
$$

where $\mathbf{1}$ in the cost function and in the right hand side are the vectors of the right dimensions made by all 1's. Then $y$ is a solution of $P(a)$ with optimal value $v^{*}$ if and only if $x / v^{*}$ is an optimal strategy for Player I.

It is clear that, when translating the game theory problem in this form, and when considering approximating games, we can only vary the matrix $P$, while the cost and the right hand side functions are fixed.

Example 1.2 (approximation of a function). Let $f, v_{1}, \ldots, v_{n} \in \mathcal{C}([\alpha, \beta]), \alpha<\beta$. We are interested in approximating a function $f$ by a linear combination of our data functions $v_{1}, \ldots, v_{n}$. We consider this approximation problem under two criteria:

(a) One-sided $L_{1}$ approximation.

Let $x \in \mathbb{R}^{n}$ be such that

$$
\sum_{i=1}^{n} v_{i}(t) x_{i} \geq f(t), \quad \text { for all } t \in[\alpha, \beta]
$$


Then

$$
\begin{gathered}
\left\|f-\sum_{i=1}^{n} x_{i} v_{i}\right\|_{1}=\int_{\alpha}^{\beta}\left[\sum_{i=1}^{n} v_{i}(t) x_{i}-f(t)\right] d t \\
=\sum_{i=1}^{n}\left(\int_{\alpha}^{\beta} v_{i}(t) d t\right) x_{i}-\int_{\alpha}^{\beta} f(t) d t .
\end{gathered}
$$

Setting

$$
c_{i}=\int_{\alpha}^{\beta} v_{i}(t) d t, \quad i=1, \ldots, n,
$$

we obtain readily that the best $L_{1}$-approximation from above to $f$ is $\sum_{i=1}^{n} \bar{x}_{i} v_{i}$, where $\bar{x} \in \mathbb{R}^{n}$ is an optimal solution of the semi-infinite problem

$$
\begin{array}{rll}
P_{1}: & \text { inf } & c^{\prime} x \\
& \text { s.t. } & \sum_{i=1}^{n} v_{i}(t) x_{i} \geq f(t), t \in[\alpha, \beta] .
\end{array}
$$

The feasible set of the problem $P_{1}$ coincides with the feasible set of $P(a)$ by taking $T=[\alpha, \beta]$, $a_{t}=\left(v_{1}(t), \ldots, v_{n}(t)\right)$, and $b_{t}=f(t)$. Notice however that (1) links left-hand side perturbations with perturbations of the objective function.

(b) Two-sided $L_{\infty}$ uniform approximation.

In this case, a best uniform approximation to $f$ is $\sum_{i=1}^{n} \bar{x}_{i} v_{i}$, where $\bar{x} \in \mathbb{R}^{n+1}$ is an optimal solution of

$$
\begin{array}{rll}
P_{2}: & \text { inf } & x_{n+1} \\
\text { s.t. } & -x_{n+1} \leq f(s)-\sum_{i=1}^{n} v_{i}(s) x_{i} \leq x_{n+1}, s \in[\alpha, \beta] .
\end{array}
$$

The problem $P_{2}$ can be written in the form $P(a)$, by taking $T=[\alpha, \beta] \times\{0,1\}$,

$$
a_{(s, k)}=\left((-1)^{k} v_{1}(s), \ldots,(-1)^{k} v_{n}(s), 1\right), \quad b_{(s, k)}=(-1)^{k} f(s), \forall(s, k) \in T,
$$

and $c=(0, \ldots, 0,1) \in \mathbb{R}^{n+1}$, but the perturbations of $a$ are subject to certain constraints $\Omega \subset \mathcal{C}([\alpha, \beta])$ (e.g., the last component of $a_{(s, k)}$ cannot be perturbed). In this case, only sufficient conditions for the continuity properties of $\mathcal{F}, \mathcal{S}$ and $v$ could apply for the admissible perturbations.

There are antecedents to this paper, dealing with various perturbations of groups of data, but not all of them at the same time. Among them, we quote [14], dealing with the problem of generic uniqueness of solution of linear programming problems under perturbations of the matrix of the constraints, and [7] dealing with lower semicontinuity of the feasible set. Other examples are [6] and [8]. Our present work continues the analysis of [7].

The rest of this paper is organized as follows. Section 2 characterizes the sets $\operatorname{dom} \mathcal{F}, \mathcal{B}_{\mathcal{F}}, \mathcal{U}_{\mathcal{F}}$, $\operatorname{dom} \mathcal{S}, \mathcal{B}_{\mathcal{S}}$, and $\mathcal{U}_{\mathcal{S}}$ and their interiors in terms of the data (in this case $\bar{a} \in \mathcal{C}\left(T, \mathbb{R}^{n}\right)$ ). Section 3 deals with conditions for $\mathcal{F}$ to be lower semicontinuous (lsc) or upper semicontinuous (usc) at a given $\bar{a} \in \operatorname{dom} \mathcal{F}$ (observe that $\mathcal{F}$ is always closed graph). Section 4 tackles similar problems regarding $\mathcal{S}$ and $v$ (for which lower and upper semicontinuities must be understood in the sense of real extended functions). It is important to note that the conditions guaranteeing continuity properties of $\mathcal{F}, \mathcal{S}$ and $v$ at a given $\bar{a}$ are frequently expressed in terms of the membership of $\bar{a}$ to the above sets and their interiors. Finally, Section 5 compares the results in this paper (left-hand side perturbations) with those corresponding to the classical case of arbitrary perturbations. 


\section{Characterizations and stability properties}

We begin this section by introducing the necessary notation. We denote by $\mathbf{0}_{n}$ the vector of zeros and by $\|\cdot\|$ the Euclidean norm in $\mathbb{R}^{n}$. The closed unit ball and the distance associated to the above norm are denoted by $B\left(\mathbf{0}_{n} ; 1\right)$ and $d$, respectively. Given $A \subset \mathbb{R}^{n}$, int $A, \operatorname{cl} A$ or $\bar{A}$, bd $A, \operatorname{span} A$, aff $A$ and conv $A$ denote the interior, the closure, the boundary, the linear subspace spanned by $A$, the affine manifold spanned by $A$, and the convex hull of $A$, respectively, whereas cone $A:=\mathbb{R}_{+}$conv $A$ denotes the convex conical hull of $A \cup\left\{\mathbf{0}_{n}\right\}$. We also define the normal cone of a nonempty closed set $A \subset \mathbb{R}^{n}$ at $\bar{x} \in A$ by

$$
N_{A}(\bar{x})=\left\{y \in \mathbb{R}^{n}: y^{\prime}(x-\bar{x}) \geq 0, \forall x \in A\right\} .
$$

The dimension of a convex set $A$ is denoted by $\operatorname{dim} A$ and the epigraph (respectively, hypograph) of a function $f: \mathbb{R}^{n} \rightarrow \mathbb{R} \cup\{ \pm \infty\}$ by epi $f$ (respectively, hypo $f$ ). We recall that $f$ is a lower semicontinuous convex function if and only if epi $f$ is a closed convex set. In this case, the subdifferential of $f$ at a point $\bar{x} \in \operatorname{dom} f$ is given by the formula

$$
\begin{aligned}
\partial f(\bar{x}) & =\left\{y \in \mathbb{R}^{n}:(y,-1) \in N_{\mathrm{epi} f}(\bar{x}, f(\bar{x}))\right\} \\
& =\left\{y \in \mathbb{R}^{n}: f(x)-f(\bar{x}) \geq y^{\prime}(x-\bar{x}), \forall x \in \mathbb{R}^{n}\right\} .
\end{aligned}
$$

Given a nonempty closed convex set $F$ we define the positively homogeneous functions

$$
\sigma_{F}(q)=\sup _{x \in F} q^{\prime} x \quad \text { and } \quad \tau_{F}(q)=\inf _{x \in F} q^{\prime} x
$$

Notice that hypo $\tau_{F}=-\operatorname{epi} \sigma_{F}$ and that both sets are closed convex cones of $\mathbb{R}^{n+1}$. Further, given a cone $C$ we denote by

$$
C^{\circ}=\left\{q \in \mathbb{R}^{n}: q^{\prime} x \geq 0, \forall x \in C\right\}
$$

its polar. It is known that $C \subset C^{\circ \circ}$ with equality whenever the cone $C$ is closed and convex.

Given a convex set $A$, another cone attached to it plays an important role in convexity. It is the recession cone of $A$ and it is defined as

$$
A_{\infty}=\{x: a+t x \in A \quad \forall a \in A, \forall t \geq 0\}
$$

Two convex cones associated with each $a \in \mathcal{C}\left(T, \mathbb{R}^{n}\right)$ play an important role in this paper. They are the moment and the characteristic cones of $a$, and are defined as follows:

$$
M(a):=\text { cone }\left\{a_{t}: t \in T\right\}
$$

and

$$
K(a):=\text { cone }\left\{\left(a_{t}, b_{t}\right): t \in T\right\}+\mathbb{R}_{+}\left(\mathbf{0}_{n},-1\right),
$$

respectively.

It follows readily from (4) that

$$
\mathcal{F}(a)=\left\{x \in \mathbb{R}^{n}: a_{t}^{\prime} x \geqslant b_{t}, \forall t \in T\right\}=\left\{x \in \mathbb{R}^{n}:(x,-1) \in K(a)^{\circ}\right\} .
$$


In a similar manner, in case $\mathcal{F}(a) \neq \emptyset$ we also get

$$
\mathcal{F}(a)_{\infty}=\left\{u \in \mathbb{R}^{n}: a_{t}^{\prime} u \geqslant 0, \forall t \in T\right\}=\left\{u \in \mathbb{R}^{n}:(u, 0) \in K(a)^{\circ}\right\} .
$$

The following characterization of $K(a)$ and its polar $K(a)^{\circ}$ will be used in the sequel. We include a proof for completeness.

Proposition 2.1 (Characterization of $\operatorname{cl} K(a)$ and $\left.K(a)^{\circ}\right)$. Assume $\mathcal{F}(a) \neq \emptyset$. Then

$$
\operatorname{cl} K(a)=\operatorname{hypo} \tau_{\mathcal{F}(a)}=-\operatorname{epi} \sigma_{\mathcal{F}(a)}
$$

and

$$
K(a)^{\circ}=\overline{\text { cone }}\left\{(x,-1),(u, 0), x \in \mathcal{F}(a), u \in \mathcal{F}(a)_{\infty}\right\} .
$$

Proof. Let $(u, \gamma) \in K(a)^{\circ}$. If $\gamma=0$, then $a_{t}^{\prime} u \geqslant 0, \forall t \in T$, that is $u \in \mathcal{F}(a)_{\infty}$. If $\gamma \neq 0$, then since $\mathbb{R}_{+}\left(\mathbf{0}_{n},-1\right) \subset K(a)$ we deduce that $\gamma<0$. It follows by (5) that

$$
(u, \gamma)=|\gamma|\left(\frac{u}{|\gamma|},-1\right) \in \mathbb{R}_{+}(\mathcal{F}(a) \times\{-1\}) .
$$

This shows that $K(a)^{\circ}$ is contained in the closed convex cone generated by $(\mathcal{F}(a) \times\{-1\}) \cup$ $\left(\mathcal{F}(a)_{\infty} \times\{0\}\right)$, while (5), (6) yield the opposite inclusion. Thus (8) holds.

Let us now notice that

$$
\begin{aligned}
\mathcal{F}(a) & =\left\{x \in \mathbb{R}^{n}: q^{\prime} x \geqslant \tau_{\mathcal{F}(a)}(q), \forall q \in \mathbb{R}^{n}\right\} \\
& =\left\{x \in \mathbb{R}^{n}: \sigma_{\mathcal{F}(a)}(p) \geq p^{\prime} x, \forall p \in \mathbb{R}^{n}\right\} .
\end{aligned}
$$

Indeed, " $\supset$ " in (9) follows directly from the definition of $\tau$ in (3), while " $\subset$ " follows from the Hahn-Banach theorem $\left(\mathcal{F}(a)\right.$ is closed and convex). To establish $(7)$, let $(q, \xi) \in \operatorname{hypo} \tau_{\mathcal{F}(a)}$ (i.e. $\left.\tau_{\mathcal{F}(a)}(q) \geq \xi\right)$ and notice that for any $x \in \mathcal{F}(a)$ we have

$$
(x,-1)^{\prime}(q, \xi)=q^{\prime} x-\xi \geq q^{\prime} x-\tau_{\mathcal{F}(a)}(q) \geq 0 .
$$

Similarly, for any $u \in \mathcal{F}(a)_{\infty}$ we have

$$
(u, 0)^{\prime}(q, \xi)=q^{\prime} u \geq 0 .
$$

Indeed, if the above relation (11) were not true, since $\mathbb{R}_{+} u \subset \mathcal{F}(a)$ we would have $\tau_{\mathcal{F}(a)}(q)=-\infty$ which contradicts the definition of $\xi$.

In view of (8), relations (10) and (11) show that hypo $\tau_{\mathcal{F}(a)} \subset\left[K(a)^{\circ}\right]^{\circ}=\operatorname{cl} K(a)$. For the opposite inclusion, let $(q, \xi) \in \operatorname{cl} K(a)=\left[K(a)^{\circ}\right]^{\circ}$. Then in view of (5) for any $x \in \mathcal{F}(a)$ we have $(q, \xi)^{\prime}(x,-1) \geq 0$, that is, $q^{\prime} x \geq \xi$ or equivalently $(q, \xi) \in$ hypo $\tau_{\mathcal{F}(a)}$.

Let us now fix $a \in \operatorname{dom} \mathcal{F}$ and denote by $\mathcal{H}_{a}$ the set of all hyperplanes in $\mathbb{R}^{n+1}$ supporting $\operatorname{cl} K(a)$. Notice that every such hyperplane $H \in \mathcal{H}_{a}$ should pass through $\mathbf{0}_{n+1}$ and is determined by a normal vector $(u, s) \in \mathbb{R}^{n+1}$ as follows:

$$
H=\left\{(q, \xi) \in \mathbb{R}^{n+1}:(u, s)^{\prime}(q, \xi)=0\right\} .
$$


Choosing adequately the normal vector - namely, $s<0$ or $s=0$ and $u \in \mathcal{F}(a)_{\infty} \backslash\left\{\mathbf{0}_{n}\right\}$ - we may always assume that

$$
\operatorname{cl} K(a) \subset H^{+}:=\left\{(q, \xi) \in \mathbb{R}^{n+1}:(u, s)^{\prime}(q, \xi) \geq 0\right\} .
$$

Notice in particular that the above yields

$$
(u, s) \in K(a)^{\circ}
$$

The following proposition describes more precisely the set $\mathcal{H}_{a}$.

Proposition 2.2 (Characterization of $\mathcal{H}_{a}$ ). Assume $\mathcal{F}(a) \neq \emptyset$. Then the elements of $\mathcal{H}_{a}$ are exactly the hyperplanes $H$ that are determined, in the sense of (12)-(13), by a normal vector which is either of the form

$$
(\hat{x},-1), \quad \hat{x} \in \partial \sigma_{\mathcal{F}(a)}\left(\mathbf{0}_{n}\right) \subset \mathcal{F}(a)
$$

or of the form

$$
(u, 0), \quad u \in \mathcal{F}(a)_{\infty} \backslash\left\{\mathbf{0}_{n}\right\} .
$$

Proof. Let $H$ be determined by the normal vector $(u, s) \in \mathbb{R}^{n+1} \backslash\left\{\mathbf{0}_{n+1}\right\}$. Then since $\left(\mathbf{0}_{n},-1\right) \in$ cl $K(a)$, relation (13) yields $s \leq 0$. If $s=0$, then by (13) again we get $a_{t}^{\prime} u \geq 0$ for all $t \in T$, thus $u \in \mathcal{F}(a)_{\infty} \backslash\left\{\mathbf{0}_{n}\right\}$. If $s<0$, then setting $\hat{x}=|s|^{-1} u$ we deduce that $H$ is also determined by the vector $(\hat{x},-1)$. By $(7)$ and $(13)$ we obtain that $(\hat{x},-1) \in N_{\text {epi } \sigma_{\mathcal{F}(a)}}\left(\mathbf{0}_{n}, 0\right)$, that is, $\hat{x} \in$ $-\partial \sigma_{\mathcal{F}(a)}\left(\mathbf{0}_{n}\right)$. This yields $\sigma_{\mathcal{F}(a)}(p) \geq p^{\prime} \hat{x}$, for all $p \in \mathbb{R}^{n}$, thus by $(9) \hat{x} \in \mathcal{F}(a)$. Conversely, one easily verifies that (13) holds true for all hyperplanes $H$ determined by vectors of the form (15) or $(16)$.

We say that $\widehat{x} \in \mathbb{R}^{n}$ is a Slater point of $a$ whenever $a_{t}^{\prime} \widehat{x}>b_{t}$ for all $t \in T$. In that case we say that $a$ satisfies the Slater condition (SC in short). If $\widehat{x} \in \mathbb{R}^{n}$ is a Slater point of $a$, then $\widehat{x} \in \operatorname{int} \mathcal{F}(a)$ and the converse holds whenever the constraint system $\left\{a_{t}^{\prime} x \geq b_{t}, t \in T\right\}$ does not contain the trivial inequality $\mathbf{0}_{n}^{\prime} x \geq 0$. The following are known facts about the connections among the Slater condition, the characteristic cone $K(a)$ and the optimal value $v(a)$ :

1. If $a$ satisfies SC, then $K(a)$ is closed [10, Theorem 5.3];

2. a satisfies SC if and only if there exists a hyperplane $H$ in $\mathbb{R}^{n+1}$ supporting $K(a)$ at the unique point $\mathbf{0}_{n+1}$;

3. Since, by [10, Theorem 8.1(ii)],

$$
v(a)=\sup \{\gamma:(c, \gamma) \in \operatorname{cl} K(a)\}=\tau_{\mathcal{F}(a)}(c),
$$

it follows that $v(a) \in \mathbb{R}$ if and only if $(\{c\} \times \mathbb{R}) \cap \operatorname{cl} K(a)$ is a proper half-line.

In the sequel of this section we want to identify the interior points of the sets $\mathcal{U}_{\mathcal{F}} \subset \mathcal{B}_{\mathcal{F}} \subset$ $\operatorname{dom} \mathcal{F}$ and $\mathcal{U}_{\mathcal{S}} \subset \mathcal{B}_{\mathcal{S}} \subset \operatorname{dom} \mathcal{S}$, which are those parameters for which the corresponding property (uniqueness of the feasible solution, boundedness of the feasible set, consistency, uniqueness of the optimal solution, boundedness of the optimal set, and solvability, respectively) is preserved 
by sufficiently small perturbations. To this aim, we collect below the known characterizations of four of the above sets in terms of either $M(a)$ or $K(a)$, namely:

$$
\begin{aligned}
& \operatorname{dom} \mathcal{F}=\left\{a \in \mathcal{C}\left(T, \mathbb{R}^{n}\right):\left(\mathbf{0}_{n}, 1\right) \notin \operatorname{cl} K(a)\right\}[10, \text { Corollary 3.1.1], } \\
& \mathcal{B}_{\mathcal{F}}=\left\{a \in \operatorname{dom} \mathcal{F}: M(a)=\mathbb{R}^{n}\right\}[10, \text { Theorem 9.3], } \\
& \mathcal{B}_{\mathcal{S}}=\{a \in \operatorname{dom} \mathcal{F}: c \in \operatorname{int} M(a)\}[10, \text { Theorem 8.1(vi)], } \\
& \mathcal{U}_{\mathcal{F}}=\{a \in \operatorname{dom} \mathcal{F}: \operatorname{cl} K(a) \text { is half-space }\} \text { [10, Theorem 5.13(iii)]. }
\end{aligned}
$$

Concerning $\operatorname{dom} \mathcal{F}$, the following facts are also known [7, Proposition 4.1]:

1. $\operatorname{dom} \mathcal{F}=\mathcal{C}\left(T, \mathbb{R}^{n}\right)$ if and only if $b_{t} \leq 0$ for all $t \in T$;

2. dom $\mathcal{F}$ is an open proper subset of $\mathcal{C}\left(T, \mathbb{R}^{n}\right)$ if and only if $\min _{t \in T} b_{t}>0$;

3. $\operatorname{dom} \mathcal{F}$ is closed in $\mathcal{C}\left(T, \mathbb{R}^{n}\right)$ if and only if $\operatorname{dom} \mathcal{F}=\mathcal{C}\left(T, \mathbb{R}^{n}\right)$.

The next two propositions provide characterizations of the above sets in terms of $\mathcal{H}_{a}$.

Proposition 2.3 (Characterization of $\operatorname{dom} \mathcal{F}, \mathcal{B}_{\mathcal{F}}, \mathcal{U}_{\mathcal{F}}$ ).

(A) The following are equivalent:

(i) $\mathcal{F}(a) \neq \emptyset$ (i.e. $a \in \operatorname{dom} \mathcal{F})$;

(ii) $\left(\mathbf{0}_{n}, 1\right) \notin \operatorname{cl} K(a)$;

(iii) there exists $H \in \mathcal{H}_{a}$ such that $\left(\mathbf{0}_{n}, 1\right) \notin H$.

(B) The following are equivalent:

(i) $\mathcal{F}(a) \neq \emptyset$ and bounded (i.e. $\left.a \in \mathcal{B}_{\mathcal{F}}\right)$;

(ii) $\left(\mathbf{0}_{n}, 1\right) \notin \operatorname{cl} K(a)$ and $\left(\mathbf{0}_{n},-1\right) \in \operatorname{int} K(a)$;

(iii) $\mathcal{H}_{a} \neq \emptyset$ and for all $H \in \mathcal{H}_{a}$ we have $\left(\mathbf{0}_{n}, 1\right) \notin H$.

(C) The following are equivalent:

(i) $\mathcal{F}(a)$ is singleton (i.e. $\left.a \in \mathcal{U}_{\mathcal{F}}\right)$;

(ii) $\mathcal{H}_{a}=\{\hat{H}\}$ (singleton) and $\left(\mathbf{0}_{n}, 1\right) \notin \hat{H}$.

Proof. (A) Assume $(i)$ holds. Pick any $x \in \mathcal{F}(a)$. Then by (5) we deduce that $(x,-1) \in K(a)^{\circ}$, which readily yields $\left(\mathbf{0}_{n}, 1\right) \notin \operatorname{cl} K(a)$. Thus $(i)$ implies $(i i)$.

Now assume $(i i)$, i.e. $\left(0_{n}, 1\right) \notin \mathrm{cl} K(a)$. Then by the Hahn-Banach theorem, there exists a hyperplane $H$ of $\mathbb{R}^{n+1}$ determined by a normal vector $(u, s) \in \mathbb{R}^{n+1} \backslash\left\{\mathbf{0}_{n+1}\right\}$ that separates strictly the singleton $\left\{\left(\mathbf{0}_{n}, 1\right)\right\}$ from the closed convex cone $\mathrm{cl} K(a)$. This yields in particular that $s \neq 0$. With no loss of generality $s<0$, that is, relations (12), (13) hold and $\left(\mathbf{0}_{n}, 1\right) \notin H^{+}$. Thus, $H \in \mathcal{H}_{a}$ and $\left(\mathbf{0}_{n}, 1\right) \notin H$. Thus (ii) implies (iii).

Assume now (iii) holds, that is, there exists $H \in \mathcal{H}_{a}$ determined by the vector $(u, s) \in$ $\mathbb{R}^{n+1} \backslash\left\{\mathbf{0}_{n+1}\right\}$ such that $\left(\mathbf{0}_{n}, 1\right) \notin H$. The latter yields $s \neq 0$. By $(13),\left(\mathbf{0}_{n},-1\right) \in \operatorname{cl} K(a) \subset H^{+}$, whence $s<0$. It follows that the vector $\left(|s|^{-1} u,-1\right)=|s|^{-1}(u, s)$ also determines $H$ and $\left(|s|^{-1} u,-1\right) \in K(a)^{\circ}$, thus by (5) $|s|^{-1} u \in \mathcal{F}(a)$.

[NB. For the equivalence (i) $\Longleftrightarrow$ (ii) see also [10, Cor. 3.11].] 
(B) Assume (i) holds. Then by (A)[(i) $\Longrightarrow($ iii) $]$ we have $\mathcal{H}_{a} \neq \emptyset$. Pick any $H \in \mathcal{H}_{a}$ (determined by a normal vector $(u, s) \in \mathbb{R}^{n+1} \backslash\left\{\mathbf{0}_{n+1}\right\}$ so that (12), (13) hold) and assume, towards a contradiction, that $\left(\mathbf{0}_{n}, 1\right) \in H \subset H^{+}$. Since $\left(\mathbf{0}_{n},-1\right) \in \operatorname{cl} K(a) \subset H^{+}$we have $\left(\mathbf{0}_{n}, 1\right),\left(\mathbf{0}_{n},-1\right) \in H^{+}$that is, $s=0$ and $(u, 0) \in K(a)^{\circ}$, see (14). By (5) $u \in \mathcal{F}(a)_{\infty} \backslash\left\{\mathbf{0}_{n}\right\}$ that is, $\mathcal{F}(a)$ is unbounded, a contradiction. Thus $(i i i)$ holds.

Let us now assume $($ iii $)$. Then by (A) $\left[(\right.$ iii $) \Longrightarrow($ ii) $]$ we have $\left(\mathbf{0}_{n}, 1\right) \notin \operatorname{cl} K(a)$. If $\left(\mathbf{0}_{n},-1\right) \in$ $\operatorname{cl} K(a) \backslash \operatorname{int} K(a)$, then there exists $H \in \mathcal{H}_{a}$ containing the line $\mathbb{R}\left(\mathbf{0}_{n},-1\right)=\mathbb{R}\left(\mathbf{0}_{n}, 1\right)$ a contradiction. Thus $(i i)$ holds.

Finally, if (ii) holds, then by $(\mathrm{A})[(\mathrm{ii}) \Longrightarrow(\mathrm{i})]$ we have $\mathcal{F}(a) \neq \emptyset$. Further, since $\operatorname{cl} K(a)$ contains a small ball around $\left(\mathbf{0}_{n},-1\right)$, it cannot admit a "vertical" supporting hyperplane, that is, if $(u, s)$ determines a hyperplane $H \in \mathcal{H}_{a}$ then $s \neq 0$. In view of $(6), \mathcal{F}(a)_{\infty}=\left\{\mathbf{0}_{n}\right\}$ and $(i)$ holds.

[NB. For the equivalence (i) $\Longleftrightarrow$ (ii) see also [10, Theorem 9.3].]

(C) By [10, Theorem 5.13(iii)] we have $|\mathcal{F}(a)|=1$ if and only if $\operatorname{cl} K(a)$ is a half-space. The latter is equivalent to $\left|\mathcal{H}_{a}\right|=1$ and since $\mathcal{F}(a) \neq \emptyset$ the unique element $\hat{H} \in \mathcal{H}_{a}$ should satisfy $\left(\mathbf{0}_{n}, 1\right) \notin H$.

Proposition 2.4 (Characterization of $\left.\operatorname{dom} \mathcal{S}, \mathcal{B}_{\mathcal{S}}, \mathcal{U}_{\mathcal{S}}\right)$.

(A) The following are equivalent:

(i) $\mathcal{S}(a) \neq \emptyset$ (i.e. $a \in \operatorname{dom} \mathcal{S})$;

(ii) there exists $H \in \mathcal{H}_{a}$ with $(c, v(a)) \in H$ and $\left(\mathbf{0}_{n}, 1\right) \notin H$;

(B) The following are equivalent:

(i) $\mathcal{S}(a) \neq \emptyset$, bounded (i.e. $a \in \mathcal{B}_{\mathcal{S}}$ );

(ii) $a \in \operatorname{dom} \mathcal{S}$ and $\left(\mathbf{0}_{n}, 1\right) \notin H$ for all $H \in \mathcal{H}_{a}$ such that $(c, v(a)) \in H$.

(C) The following are equivalent:

(i) $\mathcal{S}(a)$ is singleton (i.e. $a \in \mathcal{U}_{\mathcal{S}}$ );

(ii) there exists a unique $H \in \mathcal{H}_{a}$ such that $(c, v(a)) \in H$ and $\left(\mathbf{0}_{n}, 1\right) \notin H$.

Proof. (A) $[(\mathrm{i}) \Longrightarrow(\mathrm{ii})]$ Recall that $v(a)=\tau_{\mathcal{F}(a)}(c)$. Let $\bar{x} \in \mathcal{S}(a)$, i.e. $c^{\prime} \bar{x}=\tau_{\mathcal{F}(a)}(c)$ or equivalently

$$
(\bar{x},-1)^{\prime}\left(c, \tau_{\mathcal{F}(a)}(c)\right)=0 .
$$

Since for all $(q, \gamma)=$ hypo $\tau_{\mathcal{F}(a)}$ we have $c^{\prime} \bar{x} \geq \tau_{\mathcal{F}(a)}(c) \geq \gamma$, it follows that

$$
(\bar{x},-1)^{\prime}(q, \gamma) \geq 0
$$

Let $\bar{H}$ be the hyperplane determined by $(\bar{x},-1)$. Then $(17)$ yields $\left(c, \tau_{\mathcal{F}(a)}(c)\right)=(c, v(a)) \in \bar{H}$ and $(7)$ yields $\operatorname{cl} K(a) \subset \bar{H}^{+}$, that is $\bar{H} \in \mathcal{H}_{a}$. Obviously $\left(\mathbf{0}_{n}, 1\right) \notin \bar{H}$ ( since $(\bar{x},-1)^{\prime}\left(\mathbf{0}_{n}, 1\right)=$ $-1 \neq 0$ ) and $(i i)$ follows.

$[($ ii $) \Longrightarrow(\mathrm{i})]$ Let $H \in \mathcal{H}_{a}$ be determined by a vector $(u, s) \in \mathbb{R}^{n+1} \backslash\left\{\mathbf{0}_{n+1}\right\}$ such that

$$
\operatorname{cl} K(a) \subset H^{+}, \quad(c, v(a))=\left(c, \tau_{\mathcal{F}(a)}(c)\right) \in H \quad \text { and } \quad\left(\mathbf{0}_{n}, 1\right) \notin H .
$$


It follows that $s<0$, and $H$ is also determined by the vector $(\hat{x},-1)$ where $\hat{x}=|s|^{-1} u$. It follows that

$$
(\hat{x},-1)^{\prime}\left(c, \tau_{\mathcal{F}(a)}(c)\right)=0
$$

or equivalently, $\hat{x} \in \mathcal{S}(a)$.

(B) Notice that (18) is equivalent to

$$
(\bar{x},-1)^{\prime}(p, \beta) \leq 0, \quad \text { for all }(p, \beta) \in \operatorname{epi} \sigma_{\mathcal{F}(a)}
$$

and holds true for every $\bar{x} \in \mathcal{F}(a)$. Further, $\bar{x} \in \mathcal{S}(a)$ if and only if (17) holds, that is,

$$
(\bar{x},-1)^{\prime}\left(-c, \sigma_{\mathcal{F}(a)}(-c)\right)=0 .
$$

Combining with (19) we deduce

$$
\bar{x} \in \mathcal{S}(a) \Longleftrightarrow(\bar{x},-1) \in N_{\text {epi } \sigma_{\mathcal{F}(a)}}\left(-c, \sigma_{\mathcal{F}(a)}(-c)\right) .
$$

We deduce, in view of $(2)$, that $\mathcal{S}(a)$ coincides with the subdifferential of the lower semicontinuous convex function $\sigma_{\mathcal{F}(a)}$ at $-c$, namely,

$$
\mathcal{S}(a)=\partial \sigma_{\mathcal{F}(a)}(-c) .
$$

Thus $\mathcal{S}(a)=\partial \sigma_{\mathcal{F}(a)}(-c)$ is bounded if and only if $-c \in$ int dom $\sigma_{\mathcal{F}(a)}$, if and only if it does not exist a "vertical" hyperplane supporting epi $\sigma_{\mathcal{F}(a)}=-\operatorname{cl} K(a)$ at the point $\left(-c, \sigma_{\mathcal{F}(a)}(-c)\right)=$ $\left(-c,-\tau_{\mathcal{F}(a)}(c)\right)$ (see $\left.(7)\right)$, or equivalently, it does not exist a "vertical" hyperplane supporting $\operatorname{cl} K(a)$ at $\left(c, \tau_{\mathcal{F}(a)}(c)\right)=(c, v(a))$. Thus, any $H \in \mathcal{H}_{a}$ such that $(c, v(a)) \in H$ is determined by a vector $(u, s)$ with $s \neq 0$ (in fact, $s>0$ ). In particular $\left(\mathbf{0}_{n}, 1\right) \notin H$.

(C) Let $a \in \operatorname{dom} \mathcal{S}$. Then, $\mathcal{S}(a)=\partial \sigma_{\mathcal{F}(a)}(-c)$ is a singleton set (i.e., the convex function $\sigma_{\mathcal{F}(a)}$ is differentiable at $\left.-c\right)$ if and only if there exists a unique nonvertical hyperplane supporting epi $\sigma_{\mathcal{F}(a)}=-\operatorname{cl} K(a)$ at $(-c,-v(a))$, i.e. there exists a unique hyperplane supporting $\mathrm{cl} K(a)$ at $(c, v(a))$ with $\left(\mathbf{0}_{n}, 1\right) \notin H$.

We approach now the characterization of $\operatorname{int} \operatorname{dom} \mathcal{F}$, int $\mathcal{B}_{\mathcal{F}}$, $\operatorname{int} \mathcal{U}_{\mathcal{F}}$, int dom $\mathcal{S}$, int $\mathcal{B}_{\mathcal{S}}$, and $\operatorname{int} \mathcal{U}_{\mathcal{S}}$

Proposition 2.5 (Characterization of int $\operatorname{dom} \mathcal{F})$. Let $\bar{a} \in \mathcal{C}\left(T, \mathbb{R}^{n}\right)$.

(A) If $b_{t} \leq 0$ for all $t \in T$, then $\bar{a} \in \operatorname{int} \operatorname{dom} \mathcal{F}$.

(B) If $b_{t}>0$ for some $t \in T$, then the following statements are equivalent:

(i) $\bar{a} \in \operatorname{int} \operatorname{dom} \mathcal{F}$;

(ii) $\bar{a}$ satisfies $S C$;

(iii) $\operatorname{dim} \mathcal{F}(\bar{a})=n$;

(iv) $\mathbf{0}_{n+1} \notin \operatorname{conv}\left\{\left(\bar{a}_{t}, b_{t}\right): t \in T\right\}$.

Proof. If $b_{t} \leq 0$ for all $t \in T$, then $\mathbf{0}_{n} \in \mathcal{F}(a)$ for all $a \in \mathcal{C}\left(T, \mathbb{R}^{n}\right)$, so that $\operatorname{dom} \mathcal{F}=\mathcal{C}\left(T, \mathbb{R}^{n}\right)$. If $b_{t}>0$ for some $t \in T$, the equivalence among $(i),(i i),(i i i)$ and $(i v)$ follows from [7, Theorems 4.2 and 4.10].

Let us emphasize the interest of conditions like $(i v)$-which are easy to verify- in contrast to conditions like $(i i)$ requiring solving a linear semi-infinite program, or (iii) that can only be verified in very specific cases. 
Proposition 2.6 (Characterization of int $\left.\mathcal{B}_{\mathcal{F}}\right)$. Let $\bar{a} \in \operatorname{int} \operatorname{dom} \mathcal{F}$. Then,

$$
\bar{a} \in \operatorname{int} \mathcal{B}_{\mathcal{F}} \Longleftrightarrow \mathbf{0}_{n} \in \operatorname{int} \operatorname{conv}\left\{\bar{a}_{t}: t \in T\right\}
$$

Proof. Assume that $\mathbf{0}_{n} \in \operatorname{int} \operatorname{conv}\left\{\bar{a}_{t}: t \in T\right\}$. Then there exists $\varepsilon>0$ such that $\mathbf{0}_{n} \in$ int conv $\left\{a_{t}: t \in T\right\}$ for all $a \in \mathcal{C}\left(T, \mathbb{R}^{n}\right)$ with $\|a-\bar{a}\|_{\infty}<\varepsilon$, in which case

$$
M(a)=\text { cone }\left\{a_{t}: t \in T\right\}=\mathbb{R}^{n}
$$

and $a \in \mathcal{B}_{\mathcal{F}}$. Hence, $\bar{a} \in \operatorname{int} \mathcal{B}_{\mathcal{F}}$.

Assume now that $\mathbf{0}_{n} \notin$ int conv $\left\{\bar{a}_{t}: t \in T\right\}$. Then either $\mathbf{0}_{n} \in \operatorname{bd} \operatorname{conv}\left\{\bar{a}_{t}: t \in T\right\}$ or $\mathbf{0}_{n} \notin$ $\operatorname{conv}\left\{\bar{a}_{t}: t \in T\right\}$. In the first case there exists a hyperplane supporting conv $\left\{\bar{a}_{t}: t \in T\right\}$ at $\mathbf{0}_{n}$ while in the second case there exists a hyperplane which strictly separates $\mathbf{0}_{n}$ and $\operatorname{conv}\left\{\bar{a}_{t}: t \in T\right\}$. So, in both cases, there exists $w \in \mathbb{R}^{n} \backslash\left\{\mathbf{0}_{n}\right\}$ such that $w^{\prime} x \geq 0$ for all $x \in \operatorname{conv}\left\{\bar{a}_{t}: t \in T\right\}$. Take $a_{t}^{\varepsilon}:=\bar{a}_{t}+\varepsilon w, t \in T$, for $\varepsilon>0$ small enough to guarantee that $a^{\varepsilon} \in \operatorname{dom} \mathcal{F}$. We have $w^{\prime} x \geq \varepsilon\|w\|^{2} \geq 0$ for all $x \in \operatorname{conv}\left\{a_{t}^{\varepsilon}: t \in T\right\}$. So, cone $\left\{a_{t}^{\varepsilon}: t \in T\right\} \subset\left\{x \in \mathbb{R}^{n}: w^{\prime} x \geq 0\right\}$ and $a^{\varepsilon} \notin \mathcal{B}_{\mathcal{F}}$. Hence, $\bar{a} \notin \operatorname{int} \mathcal{B}_{\mathcal{F}}$.

In the next result we use the fact that, given $a \in \operatorname{dom} \mathcal{F}, \mathcal{F}(a)=\left\{\mathbf{0}_{n}\right\}$ if and only if $\operatorname{cl} K(a)=\mathbb{R}^{n} \times \mathbb{R}_{-}$(see $[10$, Theorem $5.10($ ii) $)$.

Proposition 2.7 (Characterization of int $\mathcal{U}_{\mathcal{F}}$ ). Given $\bar{a} \in \operatorname{int} \operatorname{dom} \mathcal{F}$ the following statements are equivalent:

(i) $\bar{a} \in \operatorname{int} \mathcal{U}_{\mathcal{F}}$;

(ii) $\mathcal{F}(\bar{a})=\left\{\mathbf{0}_{n}\right\}$ and $\mathbf{0}_{n} \in \operatorname{int} \operatorname{conv}\left\{\bar{a}_{t}: b_{t}=0, t \in T\right\}$;

(iii) $\mathcal{F}(a)=\left\{\mathbf{0}_{n}\right\}$ in some neighborhood of $\bar{a}$.

Proof. $[(i) \Rightarrow(i i)]$ Assume that $\bar{a} \in \operatorname{int} \mathcal{U}_{\mathcal{F}}$. If there is $t \in T$ such that $b_{t}>0$, then $\bar{a}$ satisfies $\operatorname{dim} \mathcal{F}(\bar{a})=n$ (by Proposition 2.5(B)), in contradiction with $|\mathcal{F}(\bar{a})|=1$. Thus, $b_{t} \leq 0$ for all $t \in T$ and so $\mathbf{0}_{n} \in \mathcal{F}(\bar{a})$ i.e. $\mathcal{F}(\bar{a})=\left\{\mathbf{0}_{n}\right\}$ by the uniqueness assumption. Since $\bar{a} \in \operatorname{int} \mathcal{U}_{\mathcal{F}}$, we have $\mathcal{F}(a)=\left\{\mathbf{0}_{n}\right\}$ for all $a$ belonging in some neighborhood of $\bar{a}$. Hence, for any such $a$ in this neighborhood, cl $K(a)=\mathbb{R}^{n} \times \mathbb{R}_{-}$.

Reasoning by contradiction, if $\mathbf{0}_{n} \notin \operatorname{int}$ conv $\left\{\bar{a}_{t}: b_{t}=0, t \in T\right\}$, following the same argument as in the proof of Proposition 2.6, we conclude the existence of $w \in \mathbb{R}^{n} \backslash\left\{\mathbf{0}_{n}\right\}$ such that $w^{\prime} x \geq 0$ for all $x \in \operatorname{conv}\left\{\bar{a}_{t}: b_{t}=0, t \in T\right\}$. Defining $a_{t}^{\varepsilon}:=\bar{a}_{t}+\varepsilon w, t \in T$, we have $w^{\prime} x>0$ for all $x \in \operatorname{conv}\left\{a_{t}^{\varepsilon}: b_{t}=0, t \in T\right\}$.

It now follows that

$$
\mathbf{0}_{n+1} \notin \operatorname{conv}\left\{\left(a_{t}^{\varepsilon}, b_{t}\right): b_{t}=0, t \in T\right\} .
$$

Indeed, if $\mathbf{0}_{n+1} \in \operatorname{conv}\left\{\left(a_{t}^{\varepsilon}, b_{t}\right): b_{t}=0, t \in T\right\}$, we shall write $\mathbf{0}_{n+1}=\sum_{i \in I} \lambda_{i}\left(a_{t_{i}}^{\varepsilon}, 0\right), \sum_{i \in I} \lambda_{i}=1$, and $\lambda_{i}>0$ for all $i \in I$ (a finite subset of $T$ ). Multiplying by $w$ both members of $\sum_{i \in I} \lambda_{i} a_{t_{i}}^{\varepsilon}=\mathbf{0}_{n}$ we get a contradiction.

Relation (20) entails $\mathbf{0}_{n} \notin \operatorname{conv}\left\{a_{t}^{\varepsilon}: t \in T\right\}$, whence $\mathbf{0}_{n+1} \notin \operatorname{conv}\left\{\left(a_{t}^{\varepsilon}, b_{t}\right): t \in T\right\}$ and the corresponding convex cone $K\left(a^{\varepsilon}\right)$ is closed (by [15, Corollary 9.6.1]). Observe that $\operatorname{cl} K\left(a^{\varepsilon}\right)=$ $K\left(a^{\varepsilon}\right) \neq \mathbb{R}^{n} \times \mathbb{R}_{-}$since

$$
K\left(a^{\varepsilon}\right) \cap\left\{(x, 0): x \in \mathbb{R}^{n}\right\}=\text { cone }\left\{a_{t}^{\varepsilon}: b_{t}=0, t \in T\right\} \neq \mathbb{R}^{n},
$$


as a consequence of $(20)$, and consequently $\mathcal{F}\left(a^{\varepsilon}\right) \neq\left\{\mathbf{0}_{n}\right\}$. Thus $a^{\varepsilon} \notin \mathcal{U}_{\mathcal{F}}$ in contradiction with $\bar{a} \in \operatorname{int} \mathcal{U}_{\mathcal{F}}$

$[(i i) \Rightarrow($ iii $)]$ Assume now that $\mathcal{F}(\bar{a})=\left\{\mathbf{0}_{n}\right\}$ and $\mathbf{0}_{n} \in \operatorname{int}\left(\operatorname{conv}\left\{\bar{a}_{t}: b_{t}=0, t \in T\right\}\right)$. Since $\mathbf{0}_{n} \in \operatorname{int}$ cone $\left\{\bar{a}_{t}: b_{t}=0, t \in T\right\}$ there exists $\varepsilon>0$ such that $\mathbf{0}_{n} \in \operatorname{int}$ cone $\left\{a_{t}: b_{t}=0, t \in T\right\}$ for all $a \in \mathcal{C}\left(T, \mathbb{R}^{n}\right)$ such that $\|a-\bar{a}\|_{\infty}<\varepsilon$. In that case cone $\left\{a_{t}: b_{t}=0, t \in T\right\}=\mathbb{R}^{n}$ and $\operatorname{cl} K(a)=\mathbb{R}^{n} \times \mathbb{R}_{-}$. So, $\mathcal{F}(a)=\left\{\mathbf{0}_{n}\right\}$ for all $a \in \operatorname{dom} \mathcal{F}$ such that $\|a-\bar{a}\|_{\infty}<\varepsilon$ and $\bar{a} \in \operatorname{int} \mathcal{U}_{\mathcal{F}}$.

$[(i i i) \Rightarrow(i)]$ This assertion is trivial.

Remark. In general, $\mathcal{F}(\bar{a})=\left\{\mathbf{0}_{n}\right\}$ and $\mathbf{0}_{n} \in \operatorname{int}\left(\operatorname{conv}\left\{\bar{a}_{t}: t \in T\right\}\right)$ do not imply $\bar{a} \in \operatorname{int} \mathcal{U}_{\mathcal{F}}$. Indeed, consider, e.g. $n=1$, and $\left(\bar{a}_{t}, b_{t}\right)=\left(t,-t^{2}\right)$ for all $t \in T=[-1,1]$. The reader may verify that $\mathcal{F}(\bar{a})=\{0\}$, and that given $\varepsilon \in] 0, \frac{1}{2}\left[\right.$, if $a_{t}^{\varepsilon}:=\bar{a}_{t}+\varepsilon$ for $t \in T, \mathcal{F}\left(a^{\varepsilon}\right) \supset[0,4 \varepsilon]$. Nevertheless, for $T$ finite, $\mathcal{F}(\bar{a})=\left\{\mathbf{0}_{n}\right\}$ implies that $\mathbf{0}_{n} \in \operatorname{int} \operatorname{conv}\left\{\bar{a}_{t}: b_{t}=0, t \in T\right\}$ and so $\bar{a} \in \operatorname{int} \mathcal{U}_{\mathcal{F}}$.

The following result deals with the set of interior points of $\operatorname{dom} \mathcal{S}$. Before giving the precise statement, we state a simple result, that will be repeatedly used in the sequel. Its proof is quite simple and will be omitted.

Proposition 2.8. (A) Suppose $\mathcal{F}(a) \neq \emptyset$. Then $u \in 0^{+} \mathcal{F}$ if and only if $a_{t}^{\prime} x \geq 0$ for all $t \in T$. (B) Suppose $\mathcal{S}(a) \neq \emptyset$. Then $u \in \mathcal{S}_{\infty}$ if and only if

$$
a_{t}^{\prime} x \geq 0 \text { for all } t \in T, \text { and } \quad c^{\prime} u=0 \text {. }
$$

In particular

$$
\mathcal{S}(\bar{a})_{\infty}=\left[\operatorname{cone}\left\{ \pm c ; \bar{a}_{t}, t \in T\right\}\right]^{\circ},
$$

where $\left[\text { cone }\left\{ \pm c ; \bar{a}_{t}, t \in T\right\}\right]^{\circ}$ denotes the positive polar of cone $\left\{ \pm c ; \bar{a}_{t}, t \in T\right\}$.

Proposition 2.9. (i) If $\bar{a} \in \operatorname{int} \operatorname{dom} \mathcal{S}$ then $\mathcal{S}(\bar{a})$ is a nonempty bounded set.

(ii) If $\mathcal{S}(\bar{a})$ is a nonempty bounded set, then $\bar{a}$ is an interior point of $\operatorname{dom} \mathcal{S}$ in the relative topology of $\operatorname{dom} \mathcal{F}$.

Proof. $(i)$ Let $\bar{a} \in \operatorname{int} \operatorname{dom} \mathcal{S}$ and suppose that $\mathcal{S}(\bar{a})$ is unbounded. Take $u \in \mathcal{S}(\bar{a})_{\infty}$ such that $\|u\|=1$. Then from Proposition 2.8 (B) we have that $\bar{a}_{t}^{\prime} u \geq 0, t \in T$, and $c^{\prime} u=0$.

Let $a_{t}^{\varepsilon}:=\bar{a}_{t}+\varepsilon u, t \in T$. By the assumption, $a^{\varepsilon} \in \operatorname{dom} \mathcal{S}$ for $\varepsilon>0$ sufficiently small. We observe that $\left(a_{t}^{\varepsilon}\right)^{\prime} u=\bar{a}_{t}^{\prime} u+\varepsilon \geq \varepsilon>0$ for all $t \in T$. Let $\mu>0$ be such that $\left|\left(a_{t}^{\varepsilon}\right)^{\prime} c\right|=\left|\bar{a}_{t}^{\prime} c\right| \leq \mu$ for all $t \in T$.

Take $v:=u-\beta \frac{c}{\|c\|}$, with $0<\beta<\frac{\varepsilon\|c\|}{\mu}$. Then,

$$
\left(a_{t}^{\varepsilon}\right)^{\prime} v=\left(a_{t}^{\varepsilon}\right)^{\prime} u-\beta \frac{\left(a_{t}^{\varepsilon}\right)^{\prime} c}{\|c\|} \geq \varepsilon-\frac{\beta \mu}{\|c\|}>0, \text { for all } t \in T,
$$

so that $v$ is a nonzero recession direction of $\mathcal{F}\left(a^{\varepsilon}\right)$ such that $c^{\prime} v=-\beta\|c\|$. Since $v\left(a^{\varepsilon}\right)=-\infty$, $\mathcal{S}\left(a^{\varepsilon}\right)=\emptyset$ (contradiction)

(ii) Now we assume that $\mathcal{S}(\bar{a}) \neq \emptyset$ is bounded. Then, from Proposition 2.8 , we get $\mathbb{R}^{n}=$ cl cone $\left\{ \pm c ; \bar{a}_{t}, t \in T\right\}$, i.e.

$$
\mathbf{0}_{n} \in \text { int cone }\left\{ \pm c ; \bar{a}_{t}, t \in T\right\} \text {. }
$$


From (22), there exists $\delta>0$ such that $\mathbf{0}_{n} \in \operatorname{int} \operatorname{cone}\left\{ \pm c ; a_{t}, t \in T\right\}$ for any $a \in \operatorname{dom} \mathcal{F}$ such that $\|a-\bar{a}\|_{\infty}<\delta$. For such a parameter $a$ we have cone $\left\{ \pm c ; a_{t}, t \in T\right\}=\mathbb{R}^{n}$, so that $\left\{\mathbf{0}_{n}\right\}=\left[\operatorname{cone}\left\{ \pm c ; a_{t}, t \in T\right\}\right]^{\circ}$. Thus, any nonempty sublevel set of the function $x \mapsto c^{\prime} x$ in $\mathcal{F}(a)$ is bounded, i.e. $a \in \operatorname{dom} \mathcal{S}$. This completes the proof.

Proposition 2.10 (Characterization of int $\mathcal{B}_{\mathcal{S}}$ ). The following holds:

$$
\operatorname{int} \mathcal{B}_{\mathcal{S}}=\{a \in \operatorname{int} \operatorname{dom} \mathcal{F}: c \in \operatorname{int} M(a)\} .
$$

Proof. Recall that $\mathcal{B}_{\mathcal{S}}=\{a \in \operatorname{dom} \mathcal{F}: c \in \operatorname{int} M(a)\}$. The conclusion is immediate taking into account that, if $\bar{a} \in \operatorname{dom} \mathcal{F}$ satisfies $c \in \operatorname{int} M(\bar{a})=\operatorname{int}$ cone $\left\{\bar{a}_{t}, t \in T\right\}$, then $c \in \operatorname{int} M(a)=$ int cone $\left\{a_{t}, t \in T\right\}$ for all $a$ sufficiently close to $\bar{a}$.

The characterization of int $\mathcal{U}_{\mathcal{S}}$ is given in the last section, in Corollary 4.12. We also refer to [12] for a characterization of those parameters $\bar{a} \in \mathcal{C}\left(T, \mathbb{R}^{n}\right)$ such that $P(a)$ has a strongly unique solution for sufficiently small perturbations of all of the data (not only of $\bar{a}$ ). This condition is obviously sufficient for $\bar{a} \in \operatorname{int} \mathcal{U}_{\mathcal{S}}$.

\section{Stability of the feasible set}

In the following sections, we shall study properties of semicontinuity and closedness of the feasible and optimal set mappings. We recall here the necessary basic definitions. Given two topological spaces $X$ and $Y$, a set-valued mapping $\mathcal{M}: X \rightrightarrows Y$ is called lower semicontinuous at $\bar{x}$ if for every open set $O \subset Y$ such that $\mathcal{M}(\bar{x}) \cap O \neq \emptyset$, there is a neighborhood $I$ of $\bar{x}$ such that for every $x \in I$ we have $\mathcal{M}(x) \cap O \neq \emptyset$. The mapping $\mathcal{M}$ is called upper semicontinuous at $\bar{x}$ if for every open set $O \subset Y$ such that $\mathcal{M}(\bar{x}) \subset O$, there is a neighborhood $I$ of $\bar{x}$ such that for every $x \in I$ we have $\mathcal{M}(x) \subset O$. Finally, the mapping $\mathcal{M}$ is said to have a closed graph at $\bar{x}$ if for every $x_{k} \rightarrow \bar{x}$ and $y_{k} \rightarrow \bar{y}$ such that $y_{k} \in \mathcal{M}\left(x_{k}\right)$, it is $\bar{y} \in \mathcal{M}(\bar{x})$. Assuming now that $X$ and $Y$ are metric spaces, we shall also use the concepts of lower (or inner) limit for the set-valued mapping $\mathcal{M}$ at $\bar{x} \in \operatorname{dom} \mathcal{M}$,

$$
\operatorname{Li}_{x \rightarrow \bar{x}} \mathcal{M}(x):=\left\{\begin{array}{c}
y \in Y: \forall\left(x_{k}\right)_{k=1}^{\infty} \rightarrow \bar{x} \text { an associated } k_{0} \text { exists } \\
\text { such that }\left(x_{k}\right)_{k=k_{0}}^{\infty} \subset \operatorname{dom} \mathcal{M} \\
\text { and } \exists y_{k} \in \mathcal{M}\left(x_{k}\right) \forall k \geq k_{0} \text { such that } y_{k} \rightarrow y
\end{array}\right\},
$$

and of upper (or outer) limit of $\mathcal{M}$ at $\bar{x} \in \operatorname{dom} \mathcal{M}$,

$$
\operatorname{Ls}_{x \rightarrow \bar{x}} \mathcal{M}(x):=\left\{\begin{array}{c}
y \in Y: \exists\left(x_{k}\right)_{k=1}^{\infty} \rightarrow \bar{x} \text { and }\left(y^{k}\right)_{k=1}^{\infty}, y^{k} \in \mathcal{M}\left(x_{k}\right), \\
\text { such that } y^{k} \rightarrow y
\end{array}\right\} .
$$

Observe that in the context of metric spaces (which is the case in this work), $\mathcal{M}$ is lower semicontinuous at $\bar{x}$ if and only if $\mathcal{M}(\bar{x}) \subset \operatorname{Li}_{x \rightarrow \bar{x}} \mathcal{M}(x)$, while $\mathcal{M}$ has a closed graph at $\bar{x}$ if and only if $\mathcal{M}(\bar{x}) \supset \operatorname{Ls}_{x \rightarrow \bar{x}} \mathcal{M}(x)$. For more about these concepts see for instance [13, 2].

If $b_{t}>0$ for some $t \in T$, then the lower semicontinuity of $\mathcal{F}$ at $\bar{a} \in \operatorname{dom} \mathcal{F}$ is equivalent to any of the conditions (i) - (iv) in Proposition 2.5 (B) (see [7, Theorem 4.2]), and any of them implies that $\mathcal{F}_{\left.\right|_{\text {dom } \mathcal{F}}}$ is lsc at $\bar{a}$. Example 4.11 in [7] shows that the converse statement does not hold. Forthcoming Proposition 3.2 will show that, if $b_{t} \leq 0$ for all $t \in T$, then SC is still a necessary condition for the lower semicontinuity of $\mathcal{F}$ under mild conditions. This result is already known, even when we relax continuity ([7, Proposition 4.5(ii)]), but the proof below is much simpler and pops-up as a direct consequence of the following lemma. 
Lemma 3.1 (Criterium for SC). Let $\bar{a} \in \operatorname{dom} \mathcal{F}$ be such that there exist $\bar{x} \in \operatorname{Li}_{a \rightarrow \bar{a}} \mathcal{F}(a)$ and $\widehat{x} \in \mathbb{R}^{n}$ such that $\bar{x}^{\prime} \widehat{x}>0$ and $a^{\varepsilon} \in \operatorname{dom} \mathcal{F}$ for $a_{t}^{\varepsilon}:=\bar{a}_{t}-\varepsilon \widehat{x}, t \in T$, and $\varepsilon>0$ sufficiently small. Then $\bar{a}$ satisfies $S C$.

Proof. Assume that $\bar{a}$ does not satisfy SC. Then, $\mathbf{0}_{n+1} \in \operatorname{conv}\left\{\left(\bar{a}_{t}, b_{t}\right), t \in T\right\}$. Let $S \subset T, S$ finite, and $\lambda_{t}>0$ for all $t \in S$ be such that

$$
\mathbf{0}_{n+1}=\sum_{t \in S} \lambda_{t}\left(\bar{a}_{t}, b_{t}\right), \quad \sum_{t \in S} \lambda_{t}=1 .
$$

Let $\bar{x}$ and $\widehat{x}$ be two points as in the statement. Let $\varepsilon>0$ be such that $a^{\varepsilon} \in \operatorname{dom} \mathcal{F}$, with $a_{t}^{\varepsilon}:=\bar{a}_{t}-\varepsilon \widehat{x}, t \in T$. From (23), we get

$$
(-\varepsilon \widehat{x}, 0)=\sum_{t \in S} \lambda_{t}\left(\bar{a}_{t}-\varepsilon \widehat{x}, b_{t}\right) \in K\left(a^{\varepsilon}\right),
$$

so that $(-\varepsilon \widehat{x})^{\prime} x \geq 0$ for all $x \in \mathcal{F}\left(a^{\varepsilon}\right)$ by Farkas' Lemma ([10, Theorem 3.1]). Consider the halfspace $H^{-}=\left\{x \in \mathbb{R}^{n}: \widehat{x}^{\prime} x \leq 0\right\}$. We have $\mathcal{F}\left(a^{\varepsilon}\right) \subset H^{-}$for $\varepsilon>0$ small enough while $\bar{x} \notin H^{-}$. This contradicts $\bar{x} \in \mathrm{Li}_{a \rightarrow \bar{a}} \mathcal{F}(a)$.

Proposition 3.2. Let $\mathcal{F}$ be lsc at $\bar{a} \in \operatorname{int} \operatorname{dom} \mathcal{F}$. If $\mathcal{F}(\bar{a}) \neq\left\{\mathbf{0}_{n}\right\}$, then $\bar{a}$ satisfies $S C$.

Proof. Under the assumption, there will exist $\bar{x} \in \mathcal{F}(\bar{a}) \subset \operatorname{Li}_{a \rightarrow \bar{a}} \mathcal{F}(a)$ such that $\bar{x} \neq \mathbf{0}_{n}$ and, if $a_{t}^{\varepsilon}:=\bar{a}_{t}-\varepsilon \bar{x}, t \in T$, we have by assumption $a^{\varepsilon} \in \operatorname{dom} \mathcal{F}$ for $\varepsilon>0$ small enough. The conclusion is immediate from Lemma 3.1.

We now analyze the connections between the set $\mathcal{F}(\bar{a})$ and the inner limit and the outer limit of sequences $\mathcal{F}\left(a^{k}\right)$, with $a^{k} \rightarrow \bar{a}$, which are represented by $\operatorname{Li}_{k \rightarrow \infty} \mathcal{F}\left(a^{k}\right)$ and $\operatorname{Ls}_{k \rightarrow \infty} \mathcal{F}\left(a^{k}\right)$, respectively. These connections allow us getting deeper results in the analysis of the lower semicontinuity of the map $\mathcal{F}$.

The next example shows that it is possible to have existence of parameters $\bar{a} \in \operatorname{dom} \mathcal{F}$ and sequences $\left\{a^{k}\right\} \subset \operatorname{dom} \mathcal{F}$ such that $a^{k} \rightarrow \bar{a}$ as $k \rightarrow \infty$ and $\operatorname{Li}_{k \rightarrow \infty} \mathcal{F}\left(a^{k}\right)=\emptyset$.

Example 3.3. Let $\bar{a}: T=\{1,2,3,4,5\} \rightarrow \mathbb{R}^{3}$ be such that $a_{1}=(1,0,0), a_{2}=(-1,0,0), a_{3}=$ $(1,1,0), a_{4}=(1,0,1), a_{5}=(0,0,-1)$, and $b=(1,-1,2,1,0)$. Then $\mathcal{F}(\bar{a})=\left\{\left(1, x_{2}, 0\right): x_{2} \geq 1\right\}$. We associate with $k \in \mathbb{N}$ the perturbed parameter $a^{k}$ such that $a_{1}^{k}=\left(1-\frac{1}{k}, \frac{1}{k^{2}}, 0\right), a_{2}^{k}=(-1,0,0)$, $a_{3}^{k}=(1,1,0), a_{4}^{k}=(1,0,1)$, and $a_{5}^{k}=(0,0,-1)$. Since $\mathcal{F}\left(a^{k}\right)=\left\{\left(1, x_{2}, 0\right): x_{2} \geq k\right\}$ for all $k \in \mathbb{N}$, we get $\operatorname{Li}_{k \rightarrow \infty} \mathcal{F}\left(a^{k}\right)=\emptyset$. Observe that, according to [7, Proposition 4.12], $\mathcal{F}_{\mid \operatorname{dom} \mathcal{F}}$ is not lsc at $\bar{a}$ (this is also obvious from $\mathcal{F}(\bar{a}) \nsubseteq \mathrm{Li}_{a \rightarrow \bar{a}} \mathcal{F}(a)=\emptyset$ ).

Proposition 3.4. Let $\bar{a} \in \mathrm{bd} \operatorname{dom} \mathcal{F}$ be such that $\bar{a}$ and $b$ do not vanish simultaneously and suppose there exists a sequence $\left(a^{k}\right)_{k=1}^{\infty} \subset \operatorname{dom} \mathcal{F}$ converging to $\bar{a}$ such that $\operatorname{Ls}_{k \rightarrow \infty} \mathcal{F}\left(a^{k}\right)=\emptyset$. Then one of the following alternatives holds:

(i) $\bar{a} \notin \operatorname{dom} \mathcal{F}$;

(ii) $\mathcal{F}(\bar{a})$ is an unbounded set and $\operatorname{int} \mathcal{F}(\bar{a})=\emptyset$.

Proof. Assume that $(i)$ does not hold, i.e. that $\bar{a} \in \operatorname{dom} \mathcal{F}$. Notice that $\operatorname{Li}_{k \rightarrow \infty} \mathcal{F}\left(a^{k}\right)=\emptyset$ since $\operatorname{Li}_{k \rightarrow \infty} \mathcal{F}\left(a^{k}\right) \subset \operatorname{Ls}_{k \rightarrow \infty} \mathcal{F}\left(a^{k}\right)=\emptyset$. Hence, $\mathcal{F}_{\text {dom } \mathcal{F}}$ is not lsc at $\bar{a}$, so that $\operatorname{int} \mathcal{F}(\bar{a})=\emptyset$ by [7, Theorem 4.10]. Now consider $x^{k} \in \mathcal{F}\left(a^{k}\right), k=1,2, \ldots$ Then the sequence $\left(x^{k}\right)_{k=1}^{\infty}$ cannot 
have bounded subsequences, and thus we can assume (by passing possibly to a subsequence $k_{r}$ ) the existence of $u=\lim _{r \rightarrow \infty} \frac{x^{k_{r}}}{\left\|x^{k_{r}}\right\|}$. Since $\left(a_{t}^{k_{r}}\right)^{\prime} x^{k_{r}} \geq b_{t}$, it follows that $\bar{a}_{t}^{\prime} u \geq 0$. Thus $u \in \mathcal{F}(\bar{a})_{\infty} \backslash\left\{\mathbf{0}_{n}\right\}$, and the set $\mathcal{F}(\bar{a})$ is unbounded.

Proposition 3.5. Let $\bar{a} \in \operatorname{bd} \operatorname{dom} \mathcal{F}$ and suppose that $\operatorname{Li}_{k \rightarrow \infty} \mathcal{F}\left(a^{k}\right)=\emptyset$ for each sequence $\left(a^{k}\right)_{k=1}^{\infty} \subset \operatorname{dom} \mathcal{F}$ converging to $\bar{a}$, with $a^{k} \neq \bar{a}$ for all $k$. Then $\bar{a} \notin \operatorname{dom} \mathcal{F}$.

Proof. Suppose that $\bar{a} \in \operatorname{dom} \mathcal{F}$ and let $\bar{x} \in \mathcal{F}(\bar{a})$. Then $\bar{x} \neq \mathbf{0}_{n}$ (otherwise, $\operatorname{dom} \mathcal{F}=\mathcal{C}\left(T, \mathbb{R}^{n}\right)$ in contradiction with $\bar{a} \in \operatorname{bd} \operatorname{dom} \mathcal{F})$. Taking $a^{k}:=\bar{a}+\frac{1}{k} \bar{x}, k=1,2, \ldots$, we get $\bar{x} \in \mathcal{F}\left(a^{k}\right)$ for all $k$, so that $\operatorname{Li}_{k \rightarrow \infty} \mathcal{F}\left(a^{k}\right) \neq \emptyset$.

In contrast with lower semicontinuity, the upper semicontinuity of $\mathcal{F}$ has a neat characterization.

Theorem 3.6 (Characterization of usc of $\mathcal{F}) \cdot \mathcal{F}$ is usc at $\bar{a} \in \operatorname{dom} \mathcal{F}$ if and only if $\mathcal{F}(\bar{a})$ is either bounded or the whole of $\mathbb{R}^{n}$.

Proof. Thanks to [11, Corollary 5.1.1], it suffices to prove that if $\mathcal{F}$ is usc at $\bar{a} \in \operatorname{dom} \mathcal{F}$ and $\mathcal{F}(\bar{a}) \neq \mathbb{R}^{n}$, then $\mathcal{F}(\bar{a})$ is bounded.

Reasoning by contradiction, suppose that $\mathcal{F}(\bar{a})$ is unbounded. Then, there will exist $u \neq \mathbf{0}_{n}$ such that the set

$$
U:=\left\{x \in \operatorname{bd} \mathcal{F}(\bar{a}): u^{\prime} x \geq 1\right\}
$$

is unbounded. In fact, if we consider a sequence $\left(x^{k}\right)_{k=1}^{\infty} \subset$ bd $\mathcal{F}(\bar{a})$ such that $\left\|x^{k}\right\| \rightarrow \infty$ as $k \rightarrow \infty$, and w.l.o.g. we suppose that $x^{k} /\left\|x^{k}\right\| \rightarrow u$, then we shall write

$$
\lim _{k \rightarrow \infty} u^{\prime} x^{k}=\lim _{k \rightarrow \infty}\left\|x^{k}\right\|=+\infty,
$$

and this shows that $U$ is unbounded.

Now we take a sequence $\left(z^{k}\right)_{k=1}^{\infty} \subset U$ without any accumulation point.

It is clear that

$$
\left(\bar{a}_{t}+\frac{1}{k} u\right)^{\prime} z^{k} \geq b_{t}+\frac{1}{k}>b_{t}, \text { for all } t \in T,
$$

and there must exist a neighborhood $V_{k}$ of $z^{k}$ such that $V_{k} \subset B\left(z^{k} ; \frac{1}{k}\right)$ and

$$
V_{k} \subset \mathcal{F}\left(a^{k}\right), k=1,2, \ldots
$$

where $a_{t}^{k}:=\bar{a}_{t}+\frac{1}{k} u, k=1,2, \ldots$ On the other hand, as $z^{k} \in \operatorname{bd} \mathcal{F}(\bar{a})$, there will exist

$$
y^{k} \in V_{k} \backslash \mathcal{F}(\bar{a}), k=1,2, \ldots
$$

The sequence $\left(y^{k}\right)_{k=1}^{\infty}$ neither has accumulation points because $\left\|y^{k}-z^{k}\right\| \leq 1 / k, k=1,2, \ldots$ and therefore

$$
W:=\mathbb{R}^{n} \backslash\left\{y_{1}, y_{2}, \ldots\right\}
$$

is an open set such that

$$
\mathcal{F}(\bar{a}) \subset W \text { and } \mathcal{F}\left(a^{k}\right) \nsubseteq W .
$$

This contradicts the upper semicontinuity of $\mathcal{F}$ as $a^{k} \rightarrow \bar{a}$ for $k \rightarrow \infty$.

Corollary 3.7. Let $\bar{a} \in \operatorname{int} \operatorname{dom} \mathcal{F}$ not be identically zero. Then, $\bar{a} \in \operatorname{int} \mathcal{B}_{\mathcal{F}}$ if and only if $\mathcal{F}$ is usc on some neighborhood of $\bar{a}$.

Proof. Since $a \in \operatorname{dom} \mathcal{F}$ is not identically zero in some neighborhood of $\bar{a}, \mathcal{F}$ is usc at $a$ if and only if $a \in \mathcal{B}_{\mathcal{F}}$. 


\section{Stability of the optimal set and the optimal value}

This section is devoted to analyze the semicontinuity of the optimal set mapping $\mathcal{S}$ and of the optimal value function.

Proposition 4.1 (Continuity properties of the value function). Given $\bar{a} \in \mathcal{C}\left(T, \mathbb{R}^{n}\right)$ the following statements hold:

(i) If $\mathcal{F}$ is lsc at $\bar{a}$, then $v$ is usc at $\bar{a}$;

(ii) if $v$ is usc at $\bar{a} \in \operatorname{dom} \mathcal{F}$, then $\bar{a} \in \operatorname{int} \operatorname{dom} \mathcal{F}$. If, additionally, $b_{t}>0$ for some $t \in T$, then $\mathcal{F}$ is lsc at $\bar{a}$.

Proof. (i) We can assume that $\bar{a} \in \operatorname{dom} \mathcal{F}$ (otherwise $v$ is trivially usc at $\bar{a}$ ). Thus, $v(\bar{a}) \in$ $\mathbb{R} \cup\{-\infty\}$. Take an arbitrary $\mu>v(\bar{a})$.

Let $\left(a^{k}\right)_{k=1}^{\infty}$ be a sequence in $\mathcal{C}\left(T, \mathbb{R}^{n}\right)$ such that $a^{k} \rightarrow \bar{a}$ as $k \rightarrow \infty$. Let $\bar{x} \in \mathcal{F}(\bar{a})$ be such that $c^{\prime} \bar{x}<\mu$. Since the open set $V=\left\{x \in \mathbb{R}^{n}: c^{\prime} x<\mu\right\}$ satisfies $\mathcal{F}(\bar{a}) \cap V \neq \emptyset$, we have $\mathcal{F}\left(a^{k}\right) \cap V \neq \emptyset$ for $k$ large enough. For each $x^{k} \in \mathcal{F}\left(a^{k}\right) \cap V$ we have $v\left(a^{k}\right) \leq c^{\prime} x^{k}<\mu$. Thus, $v$ is usc at $\bar{a}$.

(ii) Let $\mu>v(\bar{a})$. Then there exists $\delta>0$ such that $v(a)<\mu$ for all $a \in \mathcal{C}\left(T, \mathbb{R}^{n}\right)$ such that $\|a-\bar{a}\|_{\infty}<\delta$. This implies that $\bar{a} \in \operatorname{int} \operatorname{dom} \mathcal{F}$ which is equivalent to the lower semicontinuity of $\mathcal{F}$ at $\bar{a}$ under the assumption that $b_{t}>0$ for some $t \in T$ (by Proposition 2.5).

Remark. 1) Statement $(i)$ is a particular case of so-called Maximum Theorem, which is due to C. Berge [3, pp. 115-116] (see, also, [1, Theorem 4.2.2. (1)]). We include the proof for the sake of completeness.

2) The same proof of Proposition 4.1 shows that the lower semicontinuity of $\mathcal{F}_{\left.\right|_{\text {dom } \mathcal{F}}}$ entails the upper semicontinuity of $v_{\left.\right|_{\text {dom }} \mathcal{F}}$. The converse is not true. The necessity of the additional condition $b_{t}>0$ for some $t$, in Proposition 4.1(ii), follows from Example 4.7 below.

The following proposition provides a sufficient condition for the graph closedness of $\mathcal{S}$ at $\bar{a} \in \operatorname{dom} \mathcal{S}$.

Proposition 4.2 (Closed graph of $\mathcal{S}$ ). Given $\bar{a} \in \mathcal{C}\left(T, \mathbb{R}^{n}\right)$, any of the following conditions guarantees that $\mathcal{S}$ is closed graph at $\bar{a}$ :

(i) $v$ is usc at $\bar{a}$ and $v(\bar{a}) \in \mathbb{R}$;

(ii) $\mathcal{S}(\bar{a})=\mathcal{F}(\bar{a})$.

Proof. (i) Suppose that $v$ is usc at $\bar{a}$. This implies that for every sequence $\left(a^{k}\right)_{k=1}^{\infty} \subset \mathcal{C}\left(T, \mathbb{R}^{n}\right)$ converging to $\bar{a}$, we have

$$
\lim \sup _{k \rightarrow \infty} v\left(a^{k}\right) \leq v(\bar{a}) .
$$

If $\operatorname{Ls}_{k \rightarrow \infty} \mathcal{S}\left(a^{k}\right)=\emptyset$, the inclusion $\operatorname{Ls}_{k \rightarrow \infty} \mathcal{S}\left(a^{k}\right) \subset \mathcal{S}(\bar{a})$ holds trivially. Alternatively, if $x^{0} \in \operatorname{Ls}_{k \rightarrow \infty} \mathcal{S}\left(a^{k}\right)$, there will exist subsequences $\left(a^{k_{r}}\right)_{r=1}^{\infty}$ and $x^{k_{r}} \in \mathcal{S}\left(a^{k_{r}}\right), r=1,2, \ldots$, such that $\lim _{r \rightarrow \infty} x^{k_{r}}=x^{0}$. This means

$$
\left(a_{t}^{k_{r}}\right)^{\prime} x^{k_{r}} \geq b_{t}, t \in T, r=1,2, \ldots
$$

and

$$
c^{\prime} x^{k_{r}}=v\left(a^{k_{r}}\right), r=1,2, \ldots
$$


By taking limits in $(24)$, we conclude that $x^{0} \in \mathcal{F}(\bar{a})$. Taking limits now in $(25)$ one has

$$
c^{\prime} x^{0}=\lim _{r \rightarrow \infty} c^{\prime} x^{k_{r}}=\lim _{r \rightarrow \infty} v\left(a^{k_{r}}\right) \leq \lim _{\sup } \operatorname{sum}_{k \rightarrow \infty} v\left(a^{k}\right) \leq v(\bar{a})
$$

and necessarily $x^{0} \in \mathcal{S}(\bar{a})$. Consequently,

$$
\operatorname{Ls}_{a \rightarrow \bar{a}} \mathcal{S}(a) \subset \mathcal{S}(\bar{a})
$$

i.e. $\mathcal{S}$ is closed graph at $\bar{a}$.

(ii) Assume that $\mathcal{S}(\bar{a})=\mathcal{F}(\bar{a})$. Take sequences $\left(a^{k}\right)_{k=1}^{\infty}$ in $\operatorname{dom} \mathcal{S}$ and $x^{k} \in \mathcal{S}\left(a^{k}\right), k=1,2, \ldots$, converging to $\bar{a}$ and $x^{0}$ respectively. Since $\mathcal{S}\left(a^{k}\right) \subset \mathcal{F}\left(a^{k}\right), k=1,2, \ldots$, and $\mathcal{F}$ is always closed graph, one has $x^{0} \in \mathcal{F}(\bar{a})=\mathcal{S}(\bar{a})$, and we are done.

Remark Proposition $4.2(i)$, is a particular case of Theorem 4.2.1 (3) in [1].

Corollary 4.3. If $\bar{a} \in \operatorname{dom} \mathcal{S}$ and either $\mathcal{F}$ is lsc at $\bar{a}$ or $\mathcal{S}(\bar{a})=\mathcal{F}(\bar{a})$, then $\mathcal{S}$ is closed graph at $\bar{a}$.

Proof. It is a straightforward consequence of Proposition 4.1 and Proposition 4.2.

Proposition 4.4 (Characterization of lsc of the value function). Let $\bar{a} \in \mathcal{C}\left(T, \mathbb{R}^{n}\right)$ be such that $v(\bar{a}) \in \mathbb{R}$. Then, $v$ is lsc at $\bar{a}$ if and only if $\mathcal{S}(\bar{a})$ is a nonempty bounded set.

Proof. Suppose that $\mathcal{S}(\bar{a})$ is either empty or unbounded. Then the sublevel sets

$$
\left\{x \in \mathbb{R}^{n}: \bar{a}_{t}^{\prime} x \geq b_{t}, t \in T, c^{\prime} x \leq v(\bar{a})+\rho\right\}, \rho>0,
$$

are unbounded, yielding the existence of $u \in \mathbb{R}^{n}$ such that $\|u\|=1$ and $\bar{a}_{t}^{\prime} u \geq 0$ for all $t \in T$ and $c^{\prime} u=0$ (remember that $v(\bar{a}) \in \mathbb{R}$ ). Then, following the same argument that in the proof of Proposition 2.9, we establish the existence of parameters $a^{\varepsilon} \rightarrow \bar{a}$ as $\varepsilon \downarrow 0$ with $v\left(a^{\varepsilon}\right)=$ $-\infty$, so that $v$ is not lsc at $\bar{a}$. The converse statement is straightforward consequence of $[10$, Theorem 10.1].

Observe also that $v$ is lsc at $\bar{a} \in \operatorname{dom} \mathcal{F}$ if and only if $v_{\left.\right|_{\text {dom } \mathcal{F}}}$ is lsc at $\bar{a}$ because $v$ is identically $+\infty$ outside $\operatorname{dom} \mathcal{F}$.

Example 4.5. Consider the problem in $\mathbb{R}^{2}$

$$
P(\bar{a}): \quad \inf x_{2} \quad \text { s.t. } \quad t^{2} x_{1}+x_{2} \geq 2 t, t \in[0,1] .
$$

It can be realized that $\mathcal{F}(\bar{a})=$ epi $h$, where $h\left(x_{1}\right)=-x_{1}+2$ if $x_{1} \leq 1$, and $h\left(x_{1}\right)=\frac{1}{x_{1}}$ if $x>1$. Then, $v(\bar{a})=0$ and $\mathcal{S}(\bar{a})=\emptyset$. Since $\bar{a}$ satisfies SC (take, e.g., $\widehat{x}=(2,2)), \mathcal{F}$ is lsc at $\bar{a}$ and $\bar{a} \in \operatorname{int} \operatorname{dom} \mathcal{F}$. Let

$$
a_{t}^{k}=\left\{\begin{array}{cc}
\left(\frac{1}{k^{2}}, 1\right), & t \in\left[0, \frac{1}{k}\right], \\
\left(t^{2}, 1\right), & t \in\left[\frac{1}{k}, 1\right],
\end{array}\right.
$$

for each $k \in \mathbb{N}$. We have $a^{k} \in \mathcal{C}\left([0,1], \mathbb{R}^{n}\right)$ and $a^{k} \rightarrow \bar{a}$ as $k \rightarrow \infty$. Since $v\left(a^{k}\right)=-\infty$ for all $k \in \mathbb{N}, v$ is usc but not lsc at $\bar{a}$. 
Theorem 4.6 (Characterization of continuity of the value function). Assume the existence of $t \in T$ such that $b_{t}>0$. Let $\bar{a} \in \mathcal{C}\left(T, \mathbb{R}^{n}\right)$ be such that $v(\bar{a}) \in \mathbb{R}$. Then

$$
v \text { is continuous at } \bar{a} \Leftrightarrow \mathcal{S}(\bar{a}) \neq \emptyset \text { is bounded and } \bar{a} \in \operatorname{int} \operatorname{dom} \mathcal{F} \text {. }
$$

Proof. If $v$ is continuous at $\bar{a}$, then $\bar{a} \in \operatorname{int} \operatorname{dom} \mathcal{F}$ and $\mathcal{F}$ is lsc at $\bar{a}$ by Proposition 4.1. Moreover, $\mathcal{S}(\bar{a})$ is a bounded set by Proposition 4.4. The argument is reversible.

Example 4.7. Let $T=\{1,2,3,4\}, n=2$, let $a_{1}=(1,0), a_{2}=(1,-1), a_{3}=(-1,1), a_{4}=(2,-2)$. Finally, let $c=(0,1) b=(0,0,0,0)$. Then $v(a)=0$ and $\mathcal{F}(a)=\{(x, x): x \geq 0\}$. Let $a_{1}^{\epsilon}=(1,0)$, $a_{2}^{\epsilon}=(1,-1), a_{3}^{\epsilon}=(-1,1), a_{4}^{\epsilon}=(2,-2(1+\epsilon))$. Then $v\left(a^{\epsilon}\right)=0$ and $\mathcal{F}\left(a^{\epsilon}\right)=\{(0,0)\}$, and this shows that $\mathcal{F}$ is nor lsc at $a$, even with $v$ continuous at $a$.

Corollary 4.8. Assume that $v$ is finite-valued in a neighborhood of $\bar{a} \in \mathcal{C}\left(T, \mathbb{R}^{n}\right)$. Then, $\bar{a} \in$ int $\mathcal{B}_{\mathcal{S}}$ if and only if $v$ is lsc on some neighborhood of $\bar{a}$. If, additionally, there exists $t \in T$ such that $b_{t}>0$, then, $\bar{a} \in \operatorname{int} \mathcal{B}_{\mathcal{S}}$ if and only if $v$ is continuous on some neighborhood of $\bar{a}$.

Proof. It is straightforward from Proposition 4.4 and Theorem 4.6.

Observe that Example 4.7 also shows that the converse of Corollary 4.3 fails, since $\mathcal{S}$ is constant in a neighborhood of $\bar{a}$ but $\mathcal{F}$ is not lsc at $\bar{a}$ and $\mathcal{S}(\bar{a}) \neq \mathcal{F}(\bar{a})$.

Proposition 4.9. Let $\bar{a} \in \operatorname{int} \operatorname{dom} \mathcal{F}$ be such that $\mathcal{S}(\bar{a})$ is a nonempty bounded set. If there exists $t \in T$ such that $b_{t}>0$, then $\mathcal{S}$ is usc at $\bar{a}$.

Proof. Suppose that $S$ is not equibounded around $\bar{a}$. Let $\mathcal{S}(\bar{a}) \subset B\left(\mathbf{0}_{n}, k_{0}\right), k_{0} \in \mathbb{N}$. Then for each $k \geq k_{0}$ there exists $a^{k} \in \mathcal{C}\left(T, \mathbb{R}^{n}\right)$ such that $\left\|a^{k}-\bar{a}\right\|_{\infty}<\frac{1}{k}$ and $x^{k} \in \mathcal{S}\left(a^{k}\right)$ such that $\left\|x^{k}\right\| \geq k$. Due to the continuity of $v$ at $\bar{a}$ (Theorem 4.6), $v\left(a^{k}\right) \rightarrow v(\bar{a})$.

We can assume w.l.o.g. that $\frac{x^{k}}{\left\|x^{k}\right\|} \rightarrow u$, with $\|u\|=1$. Since $\left(a_{t}^{k}\right)^{\prime} x^{k} \geq b_{t}$ for all $t \in T$, dividing by $\left\|x^{k}\right\|$ and taking limits as $k \rightarrow \infty$ we get $\bar{a}_{t}^{\prime} u \geq 0$ for all $t \in T$. So, on the one hand, $u \in \mathcal{F}(\bar{a})_{\infty}$. On the other hand, from $c^{\prime} x^{k}=v\left(a^{k}\right)$, dividing again by $\left\|x^{k}\right\|$ and taking limits as $k \rightarrow \infty$, we get $c^{\prime} u=0$. Thus $u \in \mathcal{S}(\bar{a})_{\infty}$ (contradiction).

Moreover $\mathcal{S}$ is closed graph at $\bar{a}$ as a consequence of applying consecutively Proposition 4.1 $(i)$ and Proposition 4.2(i), taking into account that the assumptions imply that $\mathcal{F}$ is lsc at $\bar{a}$. Since $S$ is equibounded around $\bar{a}$ and $\mathcal{S}$ is closed graph at $\bar{a}$, we conclude that $\mathcal{S}$ is usc at $\bar{a}$ (apply, for instance, Lemma 6.3.2 in [2]).

Corollary 4.10. Assume there exists $t \in T$ such that $b_{t}>0$. If $\bar{a} \in \operatorname{int} \mathcal{B}_{\mathcal{S}}$, then $\mathcal{S}$ is usc on some neighborhood of $\bar{a}$.

The last result in this paper characterizes continuity (and lower semicontinuity) of $\mathcal{S}_{\text {|dom } \mathcal{F}}$ through the uniqueness of optimal solution of the nominal problem. So, this uniqueness is a necessary condition for the lower semicontinuity of $\mathcal{S}$.

Theorem 4.11 (Characterization of continuity of $\mathcal{S}_{\left.\right|_{\text {dom } \mathcal{F}}}$ ). Given $\bar{a} \in \mathcal{C}\left(T, \mathbb{R}^{n}\right)$, the following statements are equivalent:

(i) $\mathcal{S}_{\left.\right|_{\operatorname{dom} \mathcal{F}}}$ is continuous at $\bar{a} \in \operatorname{dom} \mathcal{F}$;

(ii) $\mathcal{S}_{\left.\right|_{\operatorname{dom} \mathcal{S}}}$ is lsc at $\bar{a} \in \operatorname{dom} \mathcal{S}$;

(iii) $\mathcal{S}(\bar{a})$ is a singleton set. 
Proof. The implication $(i) \Rightarrow(i i)$ is trivial. Let us prove $(i i i) \Rightarrow(i)$. If $\mathcal{S}(\bar{a})$ is a singleton and $W$ is an open set such that $\mathcal{S}(\bar{a}) \cap W \neq \emptyset$, obviously $\mathcal{S}(\bar{a}) \subset W$. Now we have that $\mathcal{F}_{\left.\right|_{\text {dom } \mathcal{F}}}$ is lsc at $\bar{a}$ by applying Proposition 4.5(i) in [7]. The remark after Proposition 4.1 establishes the upper semicontinuity of $v_{\left.\right|_{\text {dom } \mathcal{F}}}$, which itself implies that $\mathcal{S}_{\left.\right|_{\text {dom } \mathcal{F}}}$ is closed graph at $\bar{a}$ following the same argument that in the proof of Proposition 4.2 (i). By Theorem 10.1(ii) in [10], the boundedness of $\mathcal{S}(\bar{a})$ entails the lower semicontinuity of $v$, and so of $v_{\left.\right|_{\operatorname{dom} \mathcal{F}}}$, at $\bar{a}$. Reasoning as in Proposition 4.9 , it is easy to see that $\mathcal{S}_{\left.\right|_{\text {dom } \mathcal{F}}}$ is equibounded, and we conclude that $\mathcal{S}_{\left.\right|_{\text {dom } \mathcal{F}}}$ is usc at $\bar{a}$ by applying Lemma 6.3.2 in [2]. Therefore, $\mathcal{S}(a) \subset W$ for every $a \in \operatorname{dom} \mathcal{F}$ close enough to $\bar{a}$. By Proposition 2.9(ii), and since $\mathcal{S}(\bar{a})$ is a nonempty bounded set, $\bar{a}$ is an interior point of $\operatorname{dom} \mathcal{S}$ in the relative topology of $\operatorname{dom} \mathcal{F}$, entailing that $\mathcal{S}(a) \neq \emptyset$ if $a \in \operatorname{dom} \mathcal{F}$ is close enough to $\bar{a}$; hence, $\mathcal{S}(a) \cap W=\mathcal{S}(a) \neq \emptyset$ and $\mathcal{S}_{\text {dom } \mathcal{F}}$ is also lsc at $\bar{a}$.

We now prove that $(i i) \Rightarrow($ iii $)$ by contradiction. Let $\bar{a} \in \operatorname{dom} \mathcal{S}$, with $\mathcal{S}(\bar{a})$ being a nonsingleton closed convex set. We discuss two possible cases:

Case 1: there exist $x^{1}, x^{2} \in \mathcal{S}(\bar{a})$ such that dim span $\left\{x^{1}, x^{2}\right\}=2$.

In this case take $u \in \mathbb{R}^{n}$ such that $u^{\prime} x^{1}=0$ and $u^{\prime} x^{2}<0$. Setting $a_{t}^{\varepsilon}:=\bar{a}_{t}+\varepsilon u$ for $t \in T$, we have $x^{1} \in \mathcal{F}\left(a^{\varepsilon}\right)$ for all $\varepsilon>0$. Pick any $x^{3} \in \mathcal{S}\left(a^{\varepsilon}\right)$ if $\mathcal{S}\left(a^{\varepsilon}\right) \neq \emptyset$, or alternatively, take $x^{3} \in \mathcal{F}\left(a^{\varepsilon}\right)$ such that $c^{\prime} x^{3}<c^{\prime} x^{1}$, otherwise.

Let us prove by contradiction that $u^{\prime} x^{3} \geq 0$. Indeed, assume $u^{\prime} x^{3}<0$. Since $x^{3} \in \mathcal{F}\left(a^{\varepsilon}\right)$,

$$
\left(a_{t}^{\varepsilon}\right)^{\prime} x^{3}=\bar{a}_{t}^{\prime} x^{3}+\varepsilon u^{\prime} x^{3} \geq b_{t} \text { for all } t \in T,
$$

so that $x^{3}$ is a Slater point of $\bar{a}$. Since $x^{3} \in \mathcal{F}(\bar{a})$, the possibility $c^{\prime} x^{3}<c^{\prime} x^{1}$ is excluded and, consequently, $c^{\prime} x^{3}=c^{\prime} x^{1}$. But this is impossible as the optimal value of $\bar{a}$ would be attained at a Slater point (which is an interior point). Thus

$$
\mathcal{S}\left(a^{\varepsilon}\right) \subset H^{+}:=\left\{x \in \mathbb{R}^{n}: u^{\prime} x^{3} \geq 0\right\}
$$

while $x^{2} \notin H^{+}$. Since $x^{2} \in \mathcal{S}(\bar{a})$ we conclude that $\mathcal{S}_{\left.\right|_{\text {dom } \mathcal{S}}}$ is not lsc at $\bar{a}$.

Case 2: We now assume $\operatorname{dim} \mathcal{S}(\bar{a})=1$ with $\mathbf{0}_{n} \in$ aff $\mathcal{S}(\bar{a})$. We consider two subcases, either $\mathbf{0}_{n} \in \mathcal{S}(\bar{a})$ or $\mathbf{0}_{n} \notin \mathcal{S}(\bar{a})$.

If $\mathbf{0}_{n} \in \mathcal{S}(\bar{a})$, the previous argument applies taking $x^{1}=\mathbf{0}_{n}$ and $u=x^{2} \in \mathcal{S}(\bar{a})$, with $x^{2} \neq \mathbf{0}_{n}$. It remains to consider the case $x^{1} \in \mathcal{S}(\bar{a}), x^{1} \neq \mathbf{0}_{n}$ and for some $\lambda>1$ we have $\lambda x^{1} \in \mathcal{S}(\bar{a})$. In this case we define

$$
c_{\varepsilon}:=\alpha_{\varepsilon}\left(c+\varepsilon x^{1}\right) \text { with } \alpha_{\varepsilon}:=\frac{\|c\|}{\left\|c+\varepsilon x^{1}\right\|}
$$

for $\varepsilon \in I:=\left[0, \frac{\|c\|}{\left\|x^{1}\right\|}\left[\right.\right.$, where $c+\varepsilon x^{1} \neq \mathbf{0}_{n}$. It is evident that the functions $\varepsilon \mapsto \alpha_{\varepsilon}$ and $\varepsilon \mapsto c_{\varepsilon}$ are continuous on $I$, where we set $c:=c_{0}$.

Let $T_{\varepsilon}$ be an $n \times n$ orthogonal matrix whose entries are continuous functions of $\varepsilon$ on $I$ and such that $T_{\varepsilon} c_{\varepsilon}=c$. Obviously, $T_{0}$ is the identity $n \times n$ matrix. Associated with this transformation we introduce the parameter $a^{\varepsilon} \in \mathcal{C}\left(T, \mathbb{R}^{n}\right)$ defined as follows,

$$
a_{t}^{\varepsilon}=T_{\varepsilon} \bar{a}_{t} \forall t \in T,
$$

and its corresponding perturbed problem

$$
P\left(a^{\varepsilon}\right): \inf c^{\prime} y \quad \text { s.t. }\left(a_{t}^{\varepsilon}\right)^{\prime} y \geq b_{t}, t \in T,
$$


where we have replaced, for our convenience, the usual variable $x$ by $y$.

If $\bar{a}_{t} \neq \mathbf{0}_{n}$, one has

$$
\begin{aligned}
\left\|a_{t}^{\varepsilon}-\bar{a}_{t}\right\| & =\left\|\left(T_{\varepsilon} \bar{a}_{t}\right)-\bar{a}_{t}\right\|=\left\|\bar{a}_{t}\right\|\left\|T_{\varepsilon}\left(\frac{\bar{a}_{t}}{\left\|\bar{a}_{t}\right\|}\right)-\frac{\bar{a}_{t}}{\left\|\bar{a}_{t}\right\|}\right\| \\
& =\left\|\bar{a}_{t}\right\|\left\|T_{\varepsilon}\left(\frac{c_{\varepsilon}}{\left\|c_{\varepsilon}\right\|}\right)-\frac{c_{\varepsilon}}{\left\|c_{\varepsilon}\right\|}\right\| \\
& =\frac{\left\|\bar{a}_{t}\right\|}{\|c\|}\left\|T_{\varepsilon}\left(c_{\varepsilon}\right)-c_{\varepsilon}\right\|=\frac{\left\|\bar{a}_{t}\right\|}{\|c\|}\left\|c-c_{\varepsilon}\right\| \\
& \leq \frac{\left\|\bar{a}_{t}\right\|}{\|c\|}\left(\left|1-\alpha_{\varepsilon}\right|\|c\|+\alpha_{\varepsilon} \varepsilon\left\|x^{1}\right\|\right) \\
& \leq \frac{\mu}{\|c\|}\left(\left|1-\alpha_{\varepsilon}\right|\|c\|+\alpha_{\varepsilon} \varepsilon\left\|x^{1}\right\|\right),
\end{aligned}
$$

where $\mu:=\max _{t \in T}\left\|\bar{a}_{t}\right\|$. If $\bar{a}_{t}=\mathbf{0}_{n}$, one has $a_{t}^{\varepsilon}=\mathbf{0}_{n}$, and from the last inequality, it turns out that $a^{\varepsilon} \rightarrow \bar{a}$ uniformly as $\varepsilon \downarrow 0$.

Putting $y=T_{\varepsilon} x$ in $P\left(a^{\varepsilon}\right)$, and observing that

$$
\begin{aligned}
\left(a_{t}^{\varepsilon}\right)^{\prime} y & =\bar{a}_{t}^{\prime} T_{\varepsilon}^{\prime} y=\bar{a}_{t}^{\prime} x, \text { and } \\
c^{\prime} y & =c_{\varepsilon}^{\prime} T_{\varepsilon}^{\prime} y=c_{\varepsilon}^{\prime} x,
\end{aligned}
$$

we get an equivalent problem (orthogonal transformations preserve scalar products), with decision variables $x$ 's,

$$
\widetilde{P}\left(a^{\varepsilon}\right): \quad \inf c_{\varepsilon}^{\prime} x \quad \text { s.t. } \quad \bar{a}_{t}^{\prime} x \geq b_{t}, t \in T,
$$

whose feasible set and optimal set are $\widetilde{\mathcal{F}}\left(a^{\varepsilon}\right)=T_{\varepsilon}^{\prime}\left(\mathcal{F}\left(a^{\varepsilon}\right)\right)$ and $\widetilde{\mathcal{S}}\left(a^{\varepsilon}\right)=T_{\varepsilon}^{\prime}\left(\mathcal{S}\left(a^{\varepsilon}\right)\right)$, respectively.

Consider the open half space $H^{+}=\left\{x \in \mathbb{R}^{n}:\left(x^{1}\right)^{\prime} x>\left\|x^{1}\right\|^{2}\right\}$. We have $d\left(\lambda x^{1}, \mathbb{R}^{n} \backslash H^{+}\right)=$ $(\lambda-1)\left\|x^{1}\right\|>0$. So, by the continuity of $T_{\varepsilon}^{\prime}$ on $I$, there exists $\varepsilon_{0}>0, \varepsilon_{0}<\frac{\|c\|}{\left\|x^{1}\right\|}$, such that $d\left(T_{\varepsilon}^{\prime}\left(\lambda x^{1}\right), \mathbb{R}^{n} \backslash H^{+}\right)>\frac{(\lambda-1)\left\|x^{1}\right\|}{2}$ for all $\varepsilon \in\left[0, \varepsilon_{0}\left[\right.\right.$. Thus, the ball $W:=B\left(\lambda x^{1} ; \frac{(\lambda-1)\left\|x^{1}\right\|}{2}\right)$ satisfies $\lambda x^{1} \in W$ and $T_{\varepsilon}^{\prime}(W)=T_{\varepsilon}^{\prime}\left(\lambda x^{1}\right)+\frac{(\lambda-1)\left\|x^{1}\right\|}{2} B\left(\mathbf{0}_{n} ; 1\right) \subset H^{+}$, i.e.

$$
\left(x^{1}\right)^{\prime}\left(x-x^{1}\right)>0, \text { for all } x \in T_{\varepsilon}^{\prime}(W) \text {, for } \varepsilon \in\left[0, \varepsilon_{0}[\text {. }\right.
$$

If $\widetilde{x} \in T_{\varepsilon}^{\prime}(W) \cap \mathcal{F}(\bar{a})$, from $c^{\prime}\left(x^{1}-\widetilde{x}\right) \leq 0\left(\right.$ as $\left.x^{1} \in \mathcal{S}(\bar{a})\right)$ and $(26)$, one gets

$$
\begin{aligned}
c_{\varepsilon}^{\prime}\left(x^{1}-\widetilde{x}\right) & =\alpha_{\varepsilon}\left(c+\varepsilon x^{1}\right)^{\prime}\left(x^{1}-\widetilde{x}\right) \\
& =\alpha_{\varepsilon}\left\{c^{\prime}\left(x^{1}-\widetilde{x}\right)-\varepsilon\left(x^{1}\right)^{\prime}\left(\widetilde{x}-x^{1}\right)\right\}<0,
\end{aligned}
$$

which shows that $\widetilde{x}$ is not optimal for problem $\widetilde{P}\left(a^{\varepsilon}\right)$. So, $T_{\varepsilon}^{\prime}(W) \cap \widetilde{\mathcal{S}}\left(a^{\varepsilon}\right)=T_{\varepsilon}^{\prime}\left(W \cap \mathcal{S}\left(a^{\varepsilon}\right)\right)=\emptyset$, i.e. $W \cap \mathcal{S}\left(a^{\varepsilon}\right)=\emptyset$. On the other hand, $W \cap \mathcal{S}(\bar{a}) \neq \emptyset$ (remember that $\lambda x^{1} \in W \cap \mathcal{S}(\bar{a})$ ), and this contradicts the assumption that $\mathcal{S}_{\left.\right|_{\mathrm{dom} \mathcal{S}}}$ is lower semicontinuous at $\bar{a}$.

The proof is complete.

Corollary 4.12. The following equivalence holds: $\bar{a} \in \operatorname{int} \mathcal{U}_{\mathcal{S}}$ if and only if $\mathcal{S}$ is continuous on some neighborhood of $\bar{a}$. 


\section{$5 \quad$ Left-hand side vs. arbitrary perturbations}

As already mentioned in the introduction, stability properties of a given (nominal) optimization problem depend on the type of allowed perturbations and, even in cases where some results are identical, the corresponding proofs may be quite different. In this section we compare two models for the same problem:

$$
\begin{array}{ll}
\text { inf } & \bar{c}^{\prime} x \\
\text { s.t. } & \bar{a}_{t}^{\prime} x \geq \bar{b}_{t}, t \in T,
\end{array}
$$

where $T$ is a compact Hausdorff topological space $T, \bar{a} \in \mathcal{C}\left(T, \mathbb{R}^{n}\right), \bar{b} \in \mathcal{C}(T, \mathbb{R})$, and $\bar{c} \in$ $\mathbb{R}^{n} \backslash\left\{\mathbf{0}_{n}\right\}$. Model I (developed in this paper) considers left-hand side perturbations while Model II allows arbitrary perturbations of all the data, always preserving continuity of the coefficients with respect to the index $t \in T$. The parameters spaces are $\Theta_{1}=\mathcal{C}\left(T, \mathbb{R}^{n}\right)$, and respectively, $\Theta_{2}=\mathcal{C}\left(T, \mathbb{R}^{n}\right) \times \mathcal{C}(T, \mathbb{R}) \times \mathbb{R}^{n}$. This latter is equipped with the following metric:

$$
d\left(\left(a^{1}, b^{1}, c^{1}\right),\left(a^{2}, b^{2}, c^{2}\right)\right):=\max \left\{\left\|c^{1}-c^{2}\right\|, \max _{t \in T}\left\|\left(a_{t}^{1}, b_{t}^{1}\right)-\left(a_{t}^{2}, b_{t}^{2}\right)\right\|\right\} .
$$

The sets $\mathcal{U}_{\mathcal{F}}, \mathcal{B}_{\mathcal{F}}, \mathcal{U}_{\mathcal{S}}, \mathcal{B}_{\mathcal{S}}$, and the mappings $\mathcal{F}, \mathcal{S}: \Theta_{2} \rightrightarrows \mathbb{R}^{n}$ and $v: \Theta_{2} \rightarrow \mathbb{R} \cup\{ \pm \infty\}$ are defined in an analogous manner for both models.

\subsection{Stable properties}

Regarding Model II, almost nothing can be said for the sets $\operatorname{dom} \mathcal{F}, \operatorname{dom} \mathcal{S}, \mathcal{B}_{\mathcal{F}}$ and $\mathcal{U}_{\mathcal{F}}$ considered in Section 2. The only known relevant result is a characterization of int $\operatorname{dom} \mathcal{F}$, see [11, Theorem 5.3.3(i)]:

$$
(\bar{a}, \bar{b}, \bar{c}) \in \operatorname{int} \operatorname{dom} \mathcal{F} \Leftrightarrow \mathrm{SC} \text { holds. }
$$

The same characterization holds true for Model I whenever there exists $t \in T$ such that $\bar{b}_{t}>0$ (Proposition $2.5(\mathrm{~B})$ ). Let us observe that the interior of other sets of parameters (e.g., those providing bounded problems, inconsistent problems, etc.) has been characterized for Model II [11, Theorem 5.3.3] but not for Model I.

\subsection{Stability of the feasible set}

The characterization of the upper semicontinuity of $\mathcal{F}$ is exactly the same for both models, but the argument is much more delicate for Model I (compare proofs of Theorem 3.6 and [9, Theorem 3.1] or [5, Theorem 2.1(b)], for Models I and II, respectively).

For Model II, lower semicontinuity of $\mathcal{F}$ is equivalent to a long list of properties, e.g., $(\bar{a}, \bar{b}, \bar{c}) \in \operatorname{int} \operatorname{dom} \mathcal{F}, \mathrm{SC}$ or full dimension of $\mathcal{F}(\bar{a}, \bar{b}, \bar{c})$ ([10, Theorem 6.9] extends and improves [9, Theorem 4.1] and [5, Theorem 2.1(c)]). For Model I, however, the situation is much more complicated: according to Proposition 3.2, if $\mathcal{F}$ is lsc at $\bar{a} \in \operatorname{int} \operatorname{dom} \mathcal{F}$ and $\mathcal{F}(\bar{a}) \neq\left\{\mathbf{0}_{n}\right\}$, then $\bar{a}$ satisfies SC. Fortunately, the weaker property that $\mathcal{F}_{\left.\right|_{\text {dom } \mathcal{F}}}$ is lsc at $\bar{a}$ in Model II has a neat characterization whenever $\left(\bar{a}_{t}, \bar{b}_{t}\right) \neq \mathbf{0}_{n+1}$ for all $t \in T: \operatorname{dim} \mathcal{F}(\bar{a}) \in\{0, n\}$ ([7, Theorem 2.9]).

\subsection{Stability of the optimal set}

Classical results on Model II establish that, given $(\bar{a}, \bar{b}, \bar{c}) \in \operatorname{dom} \mathcal{S}$, the following statements hold: 
1. $\mathcal{S}$ is closed graph at $(\bar{a}, \bar{b}, \bar{c}) \Leftrightarrow$ either $\mathcal{F}$ is lsc at $(\bar{a}, \bar{b}, \bar{c})$ or $\mathcal{F}(\bar{a}, \bar{b}, \bar{c})=\mathcal{S}(\bar{a}, \bar{b}, \bar{c})[9$, Theorem 3.2];

2. let $(\bar{a}, \bar{b}, \bar{c})$ such that $\mathcal{F}(\bar{a}, \bar{b}, \bar{c})$ does not contain lines. Then, $\mathcal{S}$ is lsc at $(\bar{a}, \bar{b}, \bar{c}) \Leftrightarrow \mathcal{F}$ is lsc at $(\bar{a}, \bar{b}, \bar{c})$ and $(\bar{a}, \bar{b}, \bar{c}) \in \mathcal{U}_{\mathcal{S}}[9$, Theorem 4.2];

3. if $\mathcal{S}$ is usc at $(\bar{a}, \bar{b}, \bar{c})$, then $\mathcal{S}$ is closed graph at $(\bar{a}, \bar{b}, \bar{c})$. The converse is true whenever $(\bar{a}, \bar{b}, \bar{c}) \in \mathcal{B}_{\mathcal{S}}[9$, Theorem 3.3].

The corresponding results for Model I are less neat:

1. $\mathcal{S}$ is closed graph at $\bar{a}$ whenever either $v$ is usc at $\bar{a} \in v^{-1}(\mathbb{R})$ or $\mathcal{F}(\bar{a})=\mathcal{S}(\bar{a})$ (Proposition 4.2);

2. $\mathcal{S}$ is lsc at $\bar{a} \Rightarrow \bar{a} \in \mathcal{U}_{\mathcal{S}}$ (from Theorem 4.11);

3. If $\bar{a} \in \mathcal{B}_{\mathcal{S}} \cap \operatorname{int} \operatorname{dom} \mathcal{F}$ and $b_{t}>0$ for some $t \in T$, then $\mathcal{S}$ is usc at $\bar{a}$ (Proposition 4.9).

\subsection{Stability of the optimal value}

The stability of the optimal value for Model II is investigated in [5, Section 3] (see also [4]), which actually deals with the stability of $\left.v\right|_{v^{-1}(\mathbb{R})}$. On the other hand, Proposition 4.1 and Proposition 4.4 herein, as well as Theorem 4.6, on Model I, are concerned with $v$. Therefore, no direct comparison is in principle possible.

Moreover, some of our results in Section 4 on semicontinuity properties of $v$ can perhaps be derived by combining conveniently some results in [1] (e.g. [1, Theorems 3.1.3, 4.2.2(2), 4.2.3(1)] or Dolecki's Theorem, etc.). In this paper we provide simple and more direct proofs.

Acknowledgment. We thank the referees for their suggestions.

\section{References}

[1] Bank, B., Guddat, J., Klatte, D., Kummer, B., Tammer, K., Nonlinear Parametric Optimization, Birkhauser, Basel, 1983.

[2] Beer, G., Topologies on Closed and Closed Convex Sets, Kluwer, Dordrecht, 1993.

[3] Berge, C., Topological Spaces, Macmillan, New York, 1963.

[4] Brosowski, B., Parametric semi-infinite optimization, Methods and Procedures in Mathematical Physics 22, Verlag Peter Lang, Frankfurt am Main, 1982.

[5] Brosowski, B., Parametric semi-infinite linear programming I. Continuity of the feasible set and the optimal value, Math. Program. Study 21 (1984), 18-42.

[6] Cánovas, M.J., Dontchev, A.L., López, M.A., Parra, J., Metric regularity of semiinfinite constraint systems, Math. Program. (Ser. B), 104 (2005), 329-346. 
[7] Danillidis, A., Goberna, M.-A., Lopez, M.A., Lucchetti, R., Lower semicontinuity of the solution set mapping of linear systems relative to their domains, Set-Valued Var. Anal. 21 (2013), 67-92.

[8] Dinh, N., Goberna, M.A., López, M, On the stability of the feasible set in optimization problems, SIAM J. Optim. 20 (2010), 2254-2280.

[9] Fischer, T., Contributions to semi-infinite linear optimization. Meth. Verf. Math. Phys. 27 (1983), 175-199.

[10] Goberna, M.-A., Lopez, M.A., Linear Semi-Infinite Optimization, Wiley, Chichester, England, 1998.

[11] Goberna, M.-A., Lopez, M.A., Post-Optimal Analysis in Linear Semi-Infinite Optimization, Springer Briefs, Springer, NY, 2014.

[12] Helbig, S., Todorov, M.I., Unicity results for general linear semi-infinite optimization problems using a new concept of active constraints. Appl. Math. Optim. 38 (1998), 21-43.

[13] Lucchetti, R., Convexity and Well-Posed Problems, CMS Books in Mathematics, Springer (2006)

[14] Lucchetti, R., Radrizzani, P., Villa, S., Generic well posedness in Linear Programming, Pac. J. Optim. 4 (2008), 513-525.

[15] Rockafellar, R.T., Convex Analysis, Princeton U.P., Princeton, 1970.

[16] Todorov, M., Necessary and sufficient conditions for global lower semicontinuity in linear semi-infinite optimization, Numer. Funct. Anal. Optim. 19 (1998), 415-429.

Aris Daniilidis

DIM-CMM, UMI CNRS 2807

Blanco Encalada 2120, piso 5, Universidad de Chile

E-mail: arisd@dim.uchile.cl

http://www.dim.uchile.cl/ arisd

Research supported by the grants:

BASAL PFB-03 (Chile), FONDECYT 1130176 (Chile) and MTM2011-29064-C03-01 (Spain).

Miguel A. Goberna \& Marco A. López

Departamento de Estadística e Investigación Operativa

Universidad de Alicante, 03080, Alicante, Spain

E-mail: mgoberna@ua.es ; marco.antonio@ua.es

http://www.eio.ua.es/busqueda/infoper.asp?cod=6 ;

http://www . eio.ua.es/busqueda/infoper . asp? cod=10

Research supported by the grant MTM2014-59179-C2-1 (Spain) and the Discovery Projects DP120100467 and DP110102011 (Australian Research Council). 


\author{
Roberto Lucchetti \\ Dipartimento di Matematica. Politecnico di Milano \\ Via Bonardi 9, 20133 Milano, Italia \\ E-mail: roberto.lucchetti@polimi.it \\ http://www .mate.polimi.it/viste/pagina_personale/pagina_personale.php?id=120\&lg=en
}

Research supported by the MIUR project

"Variational and Topological Methods in the Study of Nonlinear Phenomena" (2009). 